\title{
Risk-neutral Modeling with Affine and Nonaffine Models
}

\author{
GaRLAND B. DuRHAM \\ University of Colorado
}

\begin{abstract}
Option prices provide a great deal of information regarding the market's expectations of future asset price dynamics. But, the implied dynamics are under the risk-neutral measure rather than the physical measure under which the price of the underlying asset itself evolves. This article demonstrates new techniques for joint analysis of the physical and risk-neutral models using data from both the underlying asset and options. While much of the prior work in this area has focused on affine and affine-jump models because of their analytical tractability, the techniques used in this article are straightforward to apply to a broad class of models of potential interest. The techniques are based on evaluating various integrals of interest using Monte Carlo sums over simulated volatility paths. In an application using S\&P 500 index data, we find that log volatility models perform dramatically better than affine models, but that some evidence of misspecification remains. (JEL: G13)
\end{abstract}

Option prices provide a great deal of information regarding the market's expectations of future asset price dynamics. But, the implied dynamics are under the risk-neutral measure rather than the physical measure under which the price of the underlying asset itself evolves.

This article demonstrates new techniques for joint analysis of the physical and risk-neutral models using data from both the underlying asset and options. While much of the prior work in this area has focused on affine and affine-jump

\footnotetext{
I am grateful for helpful comments and suggestions from Michael Stutzer, Magnus Dahlquist, Yang-Ho Park, Eric Renault (the editor), and two anonymous referees. Address correspondence to Garland B. Durham, Leeds School of Business, University of Colorado at Boulder, 419 UCB, Boulder, CO 80309-0419, or email: garland.durham@colorado.edu.
} 
models because of their analytical tractability, the techniques used in this article are straightforward to apply to a broad class of models of potential interest. The techniques are based on evaluating various integrals of interest using Monte Carlo sums over simulated volatility paths. Although simulation-based techniques are often computationally intensive, the approach demonstrated in this article runs in a few minutes on a typical desktop computer.

Understanding the dynamics of returns and volatility and the relationship between physical and risk-neutral measures are all fundamental issues in asset pricing. A better understanding of these issues can provide useful information regarding risk premia and help in the development of effective risk-management and hedging strategies.

The modeling framework is based on a class of stochastic volatility models that includes the possibility of jumps in both returns and volatility. In the empirical section, we examine log volatility and affine models with various jump specifications. We provide maximum likelihood estimates for the physical and riskneutral models and useful diagnostics based on generalized residuals. Although this is not novel for affine models (e.g.,Eraken 2004), the methods proposed in this article are important because they can be applied to log volatility models as well, including models with jumps in both returns and volatility.

The application uses daily observations of the S\&P 500 (SPX) and VIX indices over the period January 2, 1990 to December 29, $2006(n=4284)$. The VIX is designed to replicate a model-free measure of expected integrated volatility (IV) based on work by Britten-Iones and Neuberger 2000). Using the VIX as a proxy for IV, and given a model and candidate parameter vector, it is possible to back out the latent volatility states (under the risk-neutral measure). Given the time-series of implied volatility states and observed SPX prices, the log likelihood of the model can be computed (under the physical measure). Optimizing over the parameter space gives the maximum likelihood estimator (MLE).

Since many of the models under consideration are not nested, testing using, e.g., likelihood ratio tests is not straightforward. However, model performance can still be compared using information-based criteria such as the Akaike information criterion (AIC) or Schwarz criterion (SC). In addition, we examine several diagnostics based on generalized residuals. A useful feature is that the residuals can be decomposed into return and volatility components. The analysis of these generalized residuals proceeds along much the same lines as the residual analysis familiar from more standard time-series models. Useful graphical tools include normal-quantile plots and autocorrelation plots. Conventional model testing can be performed using, e.g., Jarque-Bera or Box-Pierce tests. These diagnostics provide a great deal of insight into what aspects of the data the models are able to fit and where they fail.

Our results corroborate previous research which finds that including jumps in returns provides a big improvement in model fit, and that including jumps in volatility as well provides an additional large improvement. However, we find that the best of the log volatility models is over 600 points in log likelihood better than 
the best affine model. Indeed, the best of the affine models is nearly 300 points worse than even the simplest log volatility model which includes jumps in neither returns nor volatility.

The diagnostics also point to serious problems in the affine models. The square-root specification for volatility of volatility simply does not reflect the data. Including jumps helps but does not resolve this problem. Moreover, using exponentially distributed jumps in volatility (as proposed by Duffie, Pan, and Singleton 2000 is problematic. This specification implies that jumps are either always positive or always negative (depending on the sign of the coefficient). But the data suggest that volatility can jump in both directions. The fitted models do a good job of capturing large positive moves in volatility, but fail to capture the large downward moves that are also observed.

Log volatility models also have difficulty with some of the diagnostics, but the defects are less severe. Although affine models are often used in applied work due to their analytical tractability, the log volatility models provide a much better description of the data.

There is a large body of related literature. A number of papers estimate the physical model directly from returns without trying to make use of any additional information on the volatility state. Lacquier, Polson, and Rossi 1994) demonstrate computationally efficient Bayesian techniques, which involve MCMC techniques for sampling over the latent state space. Jacquier, Polson, and Rossi 2004, Eraken 2001), Eraker, Johannes, and Polson 2003), Shephard and Pitt 1997), Kim, Shephard, and Chib (1998), Gallant and Tauchen 1996), Durbin and Koopman (1997), Liesenfeld and Richard (2003), Bates 2006), and Durham (2006), among many others have added to this literature. Andersen, Benzoni, and Lund 2002 and Chernov et al. 2003) provide comprehensive studies comparing a number of models using a simulated method of moments approach.

However, there has also been a great deal of work toward trying to get informationally efficient proxies for the volatility state. Such proxies are of independent interest as well as being useful in estimating models for asset returns. One particularly fruitful avenue of research is based on the idea of using highfrequency information to get a proxy for the volatility state (e.g., Andersen et al. 2003: Barndorff-Nielsen and Shephard, 2002: Ghysels, Santa-Clara, and Valkanov 2006: Garcia et al. 2011). Theory suggests that if the price process is a diffusion, then high frequency observations should provide precise information as to the volatility state. In practice, there are some problems that need to be addressed. Nonetheless, this approach shows great promise, as evidenced by the large body of recent work devoted to empirical work as well as further development of the underlying theory.

An alternative is to use the information embedded in option prices to obtain a proxy for the volatility state. The simplest way of doing so involves using BlackScholes implied volatility directly as a proxy. There are problems with this approach due to the fact that volatility is time-varying and log return distributions are non-Gaussian, contrary to the assumptions underlying the Black-Scholes pricing 
formula, but corrections are available to address these issues to some extent (e.g., Bollerslev and Zhou, 2006). More problematic is the possible presence of risk premia in the volatility dynamics. If such risk premia exist, then option prices will have different implications for spot volatility under the physical and riskneutral measures. If this distinction is ignored, then option-implied spot volatility will be systematically biased. Indeed, there is considerable evidence that this is the case (e.g. Fleming, Ostdiek, and Whaley, 1995: Christensen and Prabhalal 1998; Corrado and Millen, 2005).

The approach taken in this article uses option prices to back out implied volatility states with an explicitly specified risk-neutral measure and risk premia estimated from data. Previous works using this idea include Pastorello, Renault, and Touzi (2000), Chernov and Ghvsels 2000), Pan (2002), Jones (2003), Eraken (2004), Christoffersen, Jacobs, and Mimouni (2010), Broadie, Chernov, and Johannes 2007), among others. In theory, this approach should be capable of eliminating the bias in implied volatility found in previous empirical work, while at the same time providing direct evidence regarding risk premia. Also, since there is some overlap of the physical and risk-neutral parameters, some have argued that more informative parameter estimates for the physical model may be obtained in this manner (because of the richness of option price data). However, the theory relies heavily upon the assumption of correctly specified models.

This article builds on existing work in several directions. First, we demonstrate an efficient, simulation-based approach for inverting the risk-neutral measure to obtain the spot volatility state from a panel of observed option prices. Secondly, we demonstrate an efficient approach for maximum likelihood estimation using the observed asset prices and implied volatility states. And finally, we provide a useful set of diagnostics. The critical point is that, while much of the existing literature uses affine and affine-jump models for computational tractability, the techniques used in this article are applicable to more general models, including log volatility models that fit observed data much better.

This article is organized as follows: Section 1 describes the class of models used, Section 2 describes the methodology, Section 3 provides the application, and Section 4 concludes.

\section{MODELS}

The asset price dynamics are described by the model

$$
\begin{aligned}
d X_{t} / X_{t} & =\left[\mu_{X}\left(V_{t}\right)-\lambda_{1} \bar{\mu}_{1 t}\right] d t+\sigma_{X}\left(V_{t}\right) d W_{1 t}+J_{1 t} d N_{1 t} \\
d V_{t} & =\left[\mu_{V}\left(V_{t}\right)-\lambda_{2} \bar{\mu}_{2 t}\right] d t+\sigma_{V}\left(V_{t}\right) d W_{2 t}+J_{2 t} d N_{2 t}
\end{aligned}
$$

where $X_{t}$ is the asset price and $V_{t}$ is the volatility state, $W_{1 t}$ and $W_{2 t}$ are Brownian motions with correlation $\rho, N_{i t}$ is a Poisson process with intensity $\lambda_{i}(i=1,2)$, and $\bar{\mu}_{i t}=\mathrm{E}\left(J_{i t}\right)(i=1,2)$. 
For computational purposes, it is often more convenient to work with the log price process,

$$
\begin{aligned}
& d Y_{t}=\left[\mu_{Y}\left(V_{t}\right)-\lambda_{1 t} \bar{\mu}_{1 t}\right] d t+\sigma_{Y}\left(V_{t}\right) d W_{1 t}+\tilde{J}_{1 t} d N_{1 t} \\
& d V_{t}=\left[\mu_{V}\left(V_{t}\right)-\lambda_{2 t} \bar{\mu}_{2 t}\right] d t+\sigma_{V}\left(V_{t}\right) d W_{2 t}+J_{2 t} d N_{2 t}
\end{aligned}
$$

where $Y_{t}=\log \left(X_{t}\right), \mu_{Y}=\mu_{X}-.5 \sigma_{X}^{2}, \sigma_{Y}=\sigma_{X}$ and $\tilde{J}_{1 t}=\log \left(1+J_{1 t}\right)$. For future reference, also denote $\tilde{\mu}_{1 t}=\mathrm{E}\left(\tilde{J}_{1 t}\right)$.

The probability measure $P$ over which the model describing the dynamics of observed asset prices is defined is commonly referred to as the objective or physical measure. In order to compute option prices, we make use of an equivalent martingale measure $Q$ under which the discounted stock price, $\tilde{X}_{t}=$ $X_{t} \exp \left[\int_{0}^{t}\left(q_{\tau}-r_{\tau}\right) d \tau\right]$, is a martingale, where $q_{t}$ is the dividend payout rate and $r_{t}$ is the risk-free interest rate. $Q$ is often referred to as the risk-neutral measure. The idea is that if any derivative security with time $T$ payoff $H\left(X_{T}\right)$ is priced according to the risk-neutral expectation

$$
P_{t}=E_{t}^{Q}\left\{H\left(X_{T}\right) \exp \left[\int_{t}^{T}\left(q_{\tau}-r_{\tau}\right) d \tau\right]\right\},
$$

then there are no arbitrage opportunities (subject to some regularity conditions).

The risk neutral dynamics are given by

$$
\begin{aligned}
d X_{t} / X_{t} & =\left[\mu_{X}^{*}\left(V_{t}\right)-\lambda_{1 t} \bar{\mu}_{1 t}^{*}\right] d t+\sigma_{X}\left(V_{t}\right) d W_{1 t}^{*}+J_{1 t}^{*} d N_{1 t} \\
d V_{t} & =\left[\mu_{V}^{*}\left(V_{t}\right)-\lambda_{2 t} \bar{\mu}_{2 t}^{*}\right] d t+\sigma_{V}\left(V_{t}\right) d W_{2 t}^{*}+J_{2 t}^{*} d N_{2 t}
\end{aligned}
$$

where $W_{1}^{*}$ and $W_{2}^{*}$ are Brownian motions with correlation $\rho$ under $Q$ and $\mathrm{E}\left(J_{i t}^{*}\right)=\bar{\mu}_{i t}^{*}$ $(i=1,2)$. Similarly, the risk-neutral model for $\log$ prices is given by

$$
\begin{aligned}
& d Y_{t}=\left[\mu_{Y}^{*}\left(V_{t}\right)-\lambda_{1 t} \bar{\mu}_{1 t}^{*}\right] d t+\sigma_{Y}\left(V_{t}\right) d W_{1 t}^{*}+\tilde{J}_{1 t}^{*} d N_{1 t} \\
& d V_{t}=\left[\mu_{V}^{*}\left(V_{t}\right)-\lambda_{2 t} \bar{\mu}_{2 t}^{*}\right] d t+\sigma_{V}\left(V_{t}\right) d W_{2 t}^{*}+\tilde{J}_{2 t}^{*} d N_{2 t}
\end{aligned}
$$

where $\mu_{Y}^{*}=\mu_{X}^{*}-.5 \sigma_{X}^{2}, \tilde{J}_{1 t}^{*}=\log \left(1+\tilde{J}_{1 t}^{*}\right)$ and everything else is as above.

The relationship between physical and risk-neutral measures is well documented (e.g., Fouque, Papanicolaou, and Sircar 2000; Lewis 2000; Romano and Touzi, 1997). There are two potential sources of risk premium: diffusive risk premia are determined by the differences between $\mu_{X}\left(V_{t}\right)$ and $\mu_{X}^{*}\left(V_{t}\right)$ and between $\mu_{V}\left(V_{t}\right)$ and $\mu_{V}^{*}\left(V_{t}\right)$; and jump risk premia are determined by the differences between $J_{i t}$ and $J_{i t}^{*}(i=1,2)$. The requirement that the discounted stock price be a martingale implies that $\mu_{X}^{*}=r_{t}-q_{t}$. Given a particular specification for the physical and risk-neutral models together with a set of estimated parameters, 
Table 1 Model specifications

\section{Physical}

\section{$\log$}

$$
\begin{aligned}
& d Y_{t}=\left(r_{t}-q_{t}+\alpha_{0}+\left(\alpha_{1}-0.5\right) \exp \left(V_{t}\right)-\lambda_{1} \bar{\mu}_{1 t}\right) d t+\exp \left(V_{t} / 2\right) d W_{1 t}+\tilde{J}_{1 t} d N_{1 t} \\
& d V_{t}=\left(\kappa\left(V_{t}-\mu_{V}\right)-\lambda_{2} \bar{\mu}_{2 t}\right) d t+\sigma_{V} d W_{2 t}+J_{2 t} d N_{2 t}
\end{aligned}
$$

\section{Risk-neutral}

$$
\begin{aligned}
& d Y_{t}=\left(r_{t}-q_{t}-0.5 \exp \left(V_{t}\right)-\lambda_{1} \bar{\mu}_{1 t}\right) d t+\exp \left(V_{t} / 2\right) d W_{1 t}^{*}+\tilde{J}_{1 t}^{*} d N_{1 t} \\
& d V_{t}=\left(\kappa\left(V_{t}-\mu_{V}\right)+\eta_{1}^{*} V_{t}+\eta_{0}^{*}-\lambda_{2} \bar{\mu}_{2 t}\right) d t+\sigma_{V} d W_{2 t}^{*}+J_{2 t}^{*} d N_{2 t}
\end{aligned}
$$

\section{Affine}

Physical

$$
\begin{aligned}
& d Y_{t}=\left(r_{t}-q_{t}+\alpha_{0}+\left(\alpha_{1}-0.5\right) V_{t}-\lambda_{1} \bar{\mu}_{1 t}\right) d t+\sqrt{V_{t}} d W_{1 t}+\tilde{J}_{1 t} d N_{1 t} \\
& d V_{t}=\left(\kappa\left(V_{t}-\mu_{V}\right)-\lambda_{2} \bar{\mu}_{2 t}\right) d t+\sigma_{V} \sqrt{V_{t}} d W_{2 t} y+J_{2 t} d N_{2 t}
\end{aligned}
$$

\section{Risk-neutral}

$$
\begin{aligned}
& d Y_{t}=\left(r_{t}-q_{t}-0.5 V_{t}-\lambda_{1} \bar{\mu}_{1 t}\right) d t+\sqrt{V_{t}} d W_{1 t}^{*}+\tilde{J}_{1 t}^{*} d N_{1 t} \\
& d V_{t}=\left(\kappa\left(V_{t}-\mu_{V}\right)+\eta_{1}^{*} V_{t}+\eta_{0}^{*}-\lambda_{2} \bar{\mu}_{2 t}\right) d t+\sigma_{V} \sqrt{V_{t}} d W_{2 t}^{*}+J_{2 t}^{*} d N_{2 t}
\end{aligned}
$$

it is straightforward to back out the implied market prices of risk corresponding to the various sources of uncertainty in the model.

Both the risk-free rate, $r_{t}$, and dividend payout rate, $q_{t}$, are treated as deterministic (but time-varying). While it would be straightforward to treat these as varying randomly, the effect of doing so is negligible.

We examine two classes of models, log volatility and affine (see Table1), with several different specifications for jumps in each class.

For the log volatility models: we consider models with no jumps; jumps in returns alone; and jumps in both returns and volatility. Return jumps are either scaled by volatility or not. Let $\xi_{i t} \sim N\left(\mu_{i}, \sigma_{i}^{2}\right)(i=1,2)$. For the models with unscaled jumps, the jump distribution does not depend on the volatility state and $\tilde{J}_{1 t}=$ $\log \left(1+J_{1 t}\right)=\xi_{1 t}$, implying that $\tilde{\mu}_{1 t}=\mathrm{E}\left(\tilde{J}_{1 t}\right)=\mu_{1}$ and $\bar{\mu}_{1 t}=\mathrm{E}\left(J_{1 t}\right)=\exp \left(\mu_{1}+.5 \sigma_{1}^{2}\right)-1$. For models with scaled jumps, $\tilde{J}_{t}=\exp \left(V_{t} / 2\right) \xi_{1 t}$ with corresponding changes in $\tilde{\mu}_{1 t}$ and $\bar{\mu}_{1 t}$. Jumps in volatility either occur at the same time as jumps in returns and are correlated with them, or they are independent of jumps in returns. In either case, $J_{2 t}=\xi_{2 t}$. In the case of correlated jumps, $N_{1}=N_{2}$ and $\operatorname{corr}\left(\tilde{\xi}_{1 t}, \xi_{2 t}\right)=\rho_{J}$. In the uncorrelated case, $N_{1} \neq N_{2}$ and $\operatorname{corr}\left(\tilde{\xi}_{1 t}, \xi_{2 t}\right)=0$. Jump specifications under the risk-neutral measure are analagous in each case.

To summarize, the log volatility models under consideration are:

Log No jumps.

LogJ0 Return jumps only, unscaled.

LogJ1 Return jumps only, scaled.

LogJJ0 Jumps in returns and volatility, correlated, and unscaled. 
LogJJ1 Jumps in returns and volatility, correlated, and scaled.

LogJJ2 Jumps in returns and volatility, uncorrelated, and unscaled.

LogJJ3 Jumps in returns and volatility, uncorrelated, and scaled.

We consider an analogous collection of affine models. Let $\xi_{1 t} \sim N\left(\mu_{1}, \sigma_{1}^{2}\right)$ and $\xi_{2 t} \sim \exp (1)$ be independent random variables. In the unscaled, uncorrelated case, $\tilde{J}_{1 t}=\xi_{1 t}$ and $J_{2 t}=\mu_{2} \xi_{2 t}$. In the unscaled, correlated case, $\tilde{J}_{1 t}=\xi_{1 t}+\rho_{J} \xi_{2 t}$ and $J_{2 t}=$ $\mu_{2} \xi_{2 t}$. The scaled cases are analogous, but $\tilde{J}_{1 t}$ is scaled by a factor of $\sqrt{V_{t}}$.

To summarize, the affine models under consideration are:

Aff No jumps.

Affj0 Return jumps only, unscaled.

Affj1 Return jumps only, scaled.

AffJJ0 Jumps in returns and volatility, correlated, and unscaled.

AffJJ1 Jumps in returns and volatility, correlated, and scaled.

AffJJ2 Jumps in returns and volatility, uncorrelated, and unscaled.

AffJJ3 Jumps in returns and volatility, uncorrelated, and scaled.

AffJJ0 and AffJJ2 are essentially equivalent to the SVCJ and SVIJ models used by Eraker, Johannes, and Polson 2003).

\section{METHODS}

There are four steps involved in fitting and assessing the models under consideration:

- Given an observed panel of option prices at time $t$, invert the risk-neutral measure to get the implied volatility state.

- Given time series of observed asset prices and implied volatility states, compute the log likelihood of the data.

- Optimize over the parameter space to obtain the MLE.

- Examine some diagnostics.

The third step is straightforward. Gradients are computed numerically (the likelihood surface is smooth in the parameters). Optimization can proceed using Newton's method with a Hessian computed either using finite differences or from the outer-product of the gradient (OPG). Alternatively, a method such as BFGS that does not require Hessians could be used. In either case, standard errors can be obtained using a Hessian computed from the OPG. The candidate parameter vectors include both physical and risk-neutral parameters; implied volatility states are obtained by inverting the risk-neutral model at each evaluation of the likelihood function. See Pastorello, Patilea, and Renault (2003) for an alternative iterative approach. 
We now address each of the other steps involved in the analysis in more detail. The convention used throughout this article will be to measure time in days. Data are observed daily (weekends and holidays are ignored). Note that, in principle, there is no need for the observation frequency to be constant. This convention is maintained only for notational convenience.

\subsection{Implied Volatility}

Britten-Iones and Neubergen 2000) show that for a broad class of diffusion models, the risk-neutral expectation of the integrated variance of returns (IV hereafter) between time $t$ and time $t+\Delta$ can be computed from option prices:

$$
E_{t}^{Q} \quad{ }_{t}^{t+\Delta}\left(\frac{d X_{\tau}}{X_{\tau}}\right)^{2} d \tau=2{ }_{0}^{\infty} \frac{C(t+\Delta, K) / B_{t}(\Delta)-\max \left(0, S_{t} / B_{t}(\Delta)-K\right)}{K^{2}} d K,
$$

where $C(t+\Delta, K)$ is the price at time $t$ of a European call option with maturity $t+\Delta$ and strike price $K$ and $B_{t}(\Delta)$ is the time $t$ price of a bond paying $\$ 1$ at time $t+\Delta$. A similar expression can be constructed using put prices in place of call prices. Iiang and Tian 2005) show that the expression above is approximately true in models including jumps.

Beginning in 2003, the CBOE began reporting a revised VIX index based on implementing the right-hand side of the above expression using S\&P 500 index (SPX) options (they also provide historical data calculated using the same algorithm going back to January 2,1990). SPX options are European, so there are no issues with early exercise premia. A constant 30 -day horizon is maintained by interpolating between the integrated variances implied by options with the two shortest times to maturity greater than 8 days. The exact construction can be looked up on the $\mathrm{CBOE}$ website. The quantity actually reported is the square root of the integrated variance.

While the construction of the VIX index is intended to approximate the righthand side of the above expression, there are only a finite number of strikes traded, hence some approximation error is inevitable. The possible presence of jumps could introduce some additional approximation error. On the other hand, evidence provided by Iiang and Tian 2005) suggests that the error is likely to be quite small in practice. They argue that the VIX should provide a good proxy for the market's risk neutral expectation of the integrated volatility of returns.

While the VIX could potentially be used directly as a proxy for the spot volatility, it has been found to be a substantially biased forecaster of realized volatility. By inverting the risk-neutral measure to get the spot volatility implied by the VIX (as a proxy for expected integrated volatility), we hope that we can explain this bias by a risk premium and get better forecasts of realized volatility. 
The problem of how to obtain the value of the volatility state variable (and thus spot volatility) given an observed value of IV remains. This is comparable to the problem of inverting option prices to obtain spot volatility (e.g., Pan, 2002).

The first step involves computing the IV corresponding to a given value of the state variable. We solve this problem using simulation techniques similar to those commonly used to compute option prices (e.g., Romano and Touzi, 1997). Jiang and Tian show that

$$
E_{t}^{Q}{ }_{t}^{t+\Delta} \frac{d X_{\tau}{ }^{2}}{X_{\tau}} d \tau \approx E_{t}^{Q} \quad{ }_{t}^{t+\Delta}\left[\sigma_{Y}^{2}\left(V_{\tau}\right)+\lambda_{1} J_{1 \tau}^{*}{ }^{2}\right] d \tau .
$$

(Note that if the model does not include jumps, the above expressions are exactly equal; with jumps they are only approximately equal.) Given an initial condition $V_{t}$, it is straightforward to compute the right-hand side expectation by means of Monte Carlo techniques. Using the risk neutral model dynamics, simulate volatility paths $V_{\tau}^{(s)}$ for $\tau \in[t, t+\Delta]$ and $s=1, \ldots, S$. Then, the expectation can be approximated by

$$
E_{t}^{Q}{ }_{t}^{t+\Delta} \frac{d X_{\tau}{ }^{2}}{X_{\tau}} d \tau \approx \frac{1}{S} \sum_{s=1}^{S}{ }_{t}^{t+\Delta}\left[\sigma_{Y}^{2}\left(V_{\tau}^{(s)}\right)+\lambda_{1} \mathrm{E}\left(J_{1 \tau}^{*}{ }^{2}\right)\right] d \tau .
$$

In practice, paths are simulated using the Euler scheme with discretization $\tau_{i}=t+\delta i$ for $i=0, \ldots, \Delta / \delta$, whence

$$
E_{t}^{Q} \quad{ }_{t}^{t+\Delta} \frac{d X_{\tau}}{X_{\tau}}{ }^{2} d \tau \approx \frac{1}{S} \sum_{s=1}^{S} \sum_{i=0}^{\Delta / \delta-1}\left[\sigma_{Y}^{2}\left(V_{\tau_{i}}^{(s)}\right)+\lambda_{1} \mathrm{E}\left(J_{1, \tau_{i}}^{*}{ }^{2}\right)\right] \delta .
$$

But, what we actually need is the inverse of the mapping described above,

$$
\Gamma: I V_{t} \rightarrow V_{t} .
$$

That is, given an observed value of $I V_{t}$, we need to obtain the implied volatility state, $V_{t}$. This is accomplished using an interpolation scheme constructed as follows. Choose a grid of values, $\hat{V}_{1}, \ldots, \hat{V}_{G}$ (the hats serve to distinguish grid points from data observations). At each grid point, evaluate the integral as described above in Equation (2) to get a collection of pairs $\left\{\left(\hat{V}_{g}, \hat{V}_{g}\right), g=1, \ldots, G\right\}$. Now, we use this collection of pairs as the basis for approximating the transformation $\Gamma$. Many schemes for doing this are possible, including polynomials, splines, etc. In this article, we use a simple polynomial approximation scheme. A cubic polynomial, $\hat{\Gamma}$, is chosen to minimize $\sum_{g=1}^{G}\left[\hat{\Gamma}\left(\hat{I}_{g}\right)-\sigma_{Y}\left(\hat{V}_{g}\right)\right]^{2}$. Having found $\hat{\Gamma}$, it is easy to compute $V_{t}=\Gamma\left(I V_{t}\right) \approx \sigma_{Y}^{-1}\left(\hat{\Gamma}\left(I V_{t}\right)\right)$ for any $t$. When computing the log likelihood, we will also need to be able to compute the derivative of $\Gamma$. This is straightforward. 
The preceding procedure could also be implemented for assets other than the S\&P 500 by constructing an expected integrated volatility series directly from option prices in a manner analogous to the construction of the VIX. If options are not European, the issue of early exercise premia must be dealt with.

One could also do something similar to the above procedure using option prices directly, without going through the intermediate step of constructing the expected integrated volatility. It is straightforward to compute the mapping from spot volatility to option prices using simulation methods. The inverse transformation can be approximated using an interpolation scheme similar to that described above. Once one has started down the road of averaging over simulated volatility paths, it does not make much difference in principle whether those paths are used to compute model-implied option prices or integrated volatility.

But there are several factors that motivate using the VIX index rather than fitting option prices directly: it is less computationally costly to obtain the IV corresponding to a candidate value of the volatility state by simulating (2) than it is to simulate panels of option prices; the issue of arbitrarily choosing either a particular option price to match at each date or a weighting scheme for error minimization is avoided; and the VIX is readily available, meaning that data issues are greatly simplified. Using the VIX (or similar measures of option-implied volatility) makes doing exercises of this nature a routine matter. In particular, our results can be easily replicated by anyone interested in doing so. We believe that third-party replicability of results is important.

\subsection{Likelihood Computation}

Given observed series of log stock prices, $\mathbf{Y}=\left(Y_{1}, \ldots, Y_{n}\right)$ and integrated volatility, $\mathbf{I V}=\left(I V_{1}, \ldots, I V_{n}\right)$, the goal is to compute the $\log$ likelihood, $\log L(\mathbf{Y}, \mathbf{I V})$. In practice, this is done using the identity

$$
\begin{aligned}
\log L(\mathbf{Y}, \mathbf{I V}) & ={ }_{t=2}^{n} \log p\left(Y_{t}, I V_{t} \mid Y_{t-1}, I V_{t-1}\right) \\
& ={ }_{t=2}^{n}\left[\log p\left(Y_{t}, V_{t} \mid Y_{t-1}, V_{t-1}\right)+\log \Gamma^{\prime}\left(I V_{t}\right)\right]
\end{aligned}
$$

where $\Gamma^{\prime}\left(I V_{t}\right)$ is the Jacobian of the transformation from $I V_{t}$ to $V_{t}$ under the riskneutral model and the conditional densities, $p\left(Y_{t}, V_{t} \mid Y_{t-1}, V_{t-1}\right)$, are computed under the physical measure. Note that the risk-neutral measure is inverted at each evaluation of the likelihood to obtain the latent volatility states $V_{t}$ conditional on the candidate parameter vector.

The problem is how to compute the conditional densities. In this article, we use an Euler scheme approximation. Given a discretization, $\tau_{0}, \tau_{1}, \ldots$, where $\tau_{k+1}-\tau_{k}=\delta$ 
$(k=0,1,2, \ldots)$, the Euler scheme for the physical model is given by

$$
\begin{gathered}
Y_{\tau_{k+1}}=Y_{\tau_{k}}+\left[\mu_{Y}\left(V_{\tau_{k}}\right)-\lambda_{1} \bar{\mu}_{1 \tau_{k}}\right] \delta+\sigma_{Y}\left(V_{\tau_{k}}\right) \sqrt{\delta} \epsilon_{1, \tau_{k+1}}+{ }_{j=1}^{\eta_{1, \tau_{k+1}}} \tilde{J}_{1, \tau_{k+1}}^{(j)} \\
V_{\tau_{k+1}}=V_{\tau_{k}}+\left[\mu_{V}\left(V_{\tau_{k}}\right)-\lambda_{2} \bar{\mu}_{2 \tau_{k}}\right] \delta+\sigma_{V}\left(V_{\tau_{k}}\right) \sqrt{\delta} \epsilon_{2, \tau_{k+1}}+{ }_{j=1}^{\eta_{2, \tau_{k+1}}} J_{2, \tau_{k+1}}^{(j)}
\end{gathered}
$$

where $\epsilon_{1, \tau_{k+1}}$ and $\epsilon_{2, \tau_{k+1}}$ are standard normal with correlation $\rho ; \eta_{i, \tau_{k+1}} \sim$ Poisson $\left(\lambda_{i} \delta\right)(i=1,2)$ is the number of jumps occurring in the period $\left(\tau_{k}, \tau_{k+1}\right]$; and the sums are over the sizes of those jumps. The Euler scheme approximation for the risk-neutral model is analogous. Convergence properties are discussed in, e.g., Kloeden and Platen 1992) and Pedersen 1995).

Conditional on the volatility state at each observation date $t$, it is straightforward to calculate the innovations corresponding to the Euler approximation. For example, for LogJJ1 and setting $\delta=1$, we get

$$
\begin{aligned}
& e_{1 t}=\left(Y_{t+1}-Y_{t}-\mu_{Y}\left(V_{t}\right)+\lambda_{1}\left(\bar{\mu}_{1 t}-\tilde{\mu}_{1 t}\right)\right) / \exp \left(V_{t} / 2\right) \\
& e_{2 t}=\left(V_{t+1}-V_{t}-\mu_{V}\left(V_{t}\right)\right) / \sigma_{V},
\end{aligned}
$$

where $\bar{\mu}_{1 t}=E\left(J_{t}\right)$ and $\tilde{\mu}_{1 t}=E\left(\tilde{J}_{t}\right)$. Note that we normalize by $\exp \left(V_{t} / 2\right)$ and $\sigma_{V}$, respectively. If (3) is the data-generating process, these innovations have mean zero; but if the model includes jumps the innovations are neither normally distributed nor do they have unit variance.

The innovation distribution is a mixture of normals (mixing over the number of possible jumps) with density (again setting $\delta=1$ for simplicity)

$$
p\left(e_{1 t}, e_{2 t}\right)={ }_{j=0}^{\infty} p(j) \phi\left[\left(e_{1 t}, e_{2 t}\right)^{\prime} ; m(j), S(j)\right],
$$

where $p(j)=\exp \left(-\lambda_{1}\right) \lambda_{1}^{j} / j !, \phi(\cdot)$ denotes the normal density, and $m(j)$ and $S(j)$ have elements

$$
\begin{gathered}
m_{1}(j)=\left(j-\lambda_{1}\right) \mu_{1} \\
m_{2}(j)=\left(j-\lambda_{1}\right) \mu_{2} \\
s_{11}(j)=1+j \sigma_{1}^{2} \\
s_{22}(j)=1+j \sigma_{2}^{2} / \sigma_{V}^{2} \\
s_{21}(j)=s_{12}(j)=\rho+j \rho_{j} \sigma_{1} \sigma_{2} / \sigma_{V}
\end{gathered}
$$

In the application, we use $\delta$ equal to one day. Alternatively, one could use a finer discretization and integrate out unobserved values of the process at 
intermediate points between observations (e.g., Pedersen, 1995: Eraken, 2001: Iones, 2003: Elerian, Chib, and Shephard, 2001: Durham and Gallant, 2002, and others). While this approach is relatively straightforward for models with no jumps or with jumps in returns only, it is tedious for models with jumps in both returns and volatility since one must importance sample across both the diffusive and jump components. The results obtained using finer discretizations do not differ substantively from what is obtained using the simple Euler scheme (the one-day sample interval is already reasonably small), but there is a substantial increase in computational complexity. In contrast, the ready availability of the data and relative transparency of the methods used to obtain the results reported in this article means that it is a straightforward exercise for anyone to reproduce and confirm our findings. For the reader interested in applying the discretization approach, we have left an earlier working version of this article on our website that provides details (see also Ferriani and Pastorello 2011 for closely related work).

We truncate the series in (5) to allow a maximum of five jumps per day. Although the effect is negligible here, it is important to normalize the weights $p(j)$ of the truncated series so that they sum to one (to ensure that probability densities integrate to one).

For the affine models with correlated jumps, jumps in returns involve a sum of normal and exponential random variables. This density can be readily evaluated using standard quadrature methods.

\subsection{Diagnostics}

The availability of the log likelihood for all of the models under consideration implies that standard information-based criteria, e.g., AIC or SC, can be used for assessing the models. These are based on assessing model fit in terms of KullbackLeibler information of the data relative to the fitted model, with various penalties that depend upon the number of estimated parameters.

In practice, none of these models might be the true data-generating process. Of interest is whether they describe the data in an economically useful manner. To address this issue, we look at various diagnostics based on generalized residuals (probability integral transform). The approach is essentially the same as for standard residual analysis of commonly used time-series models.

The basic idea is as follows: Let $z_{t}$ be a sequence of random variables (possibly multivariate) with cdf $F_{t \mid t-1}\left(z_{t} \mid z_{1}, \ldots, z_{t-1}\right)$. Then, the generalized residuals are given by

$$
u_{t}=F_{t \mid t-1}\left(z_{t} \mid z_{1}, \ldots, z_{t-1}\right), \quad t=2, \ldots, n .
$$

These should be iid $U(0,1)$. The hypothesis that the $F_{t \mid t-1}$ are in fact the true cdf's of the data-generating process can thus be tested by examining diagnostics on the $u_{t}$.

It is often more convenient to first do the transformation

$$
\tilde{u}_{t}=\Phi^{-1}\left(u_{t}\right)
$$


where is the cdf of the standard normal. If the model is correct, these should be iid standard normal. Diagnostics based on distributional characteristics (e.g., normal-quantile plots or Jarque-Bera tests) or dynamics (e.g., autocorrelation plots or Box-Pierce tests) are readily available.

Although it is straightforward to compute generalized residuals corresponding to the joint cdf $F\left(Y_{t+1}, V_{t+1} \mid Y_{t}, V_{t}\right)$, it is more useful to look at the return residuals and volatility residuals separately, i.e., residuals corresponding to $F\left(Y_{t+1} \mid Y_{t}, V_{t}\right)$ and $F\left(V_{t+1} \mid V_{t}\right)$.

As with the likelihood evaluation, we evaluate cdf's using the Euler scheme with discretization interval one day. As discussed in Section 2.2 finer discretizations could also be used, but there is little difference in the results. The calculations mirror those involved in evaluating the likelihood.

\section{APPLICATION}

Data are comprised of daily observations of the SPX and VIX indices over the period January 2, 1990 to December 29, $2006(n=4284)$ downloaded from the CBOE website. The VIX is reported as annualized percentage volatility. We divide the VIX by 100 to get it in decimal form, then square the result and divide by 252 to get a measure of IV per trading day. Three-month constant maturity Treasury bill rates, obtained from the Federal Reserve website, serve as a proxy for the risk-free rate. Quarterly dividend rates for the S\&P 500 were obtained from the Standard and Poor's website. Although the model is based on expected dividends, we use actual payouts as a proxy and assume that dividends are paid out at a uniform rate over each quarter. Time is measured in trading days (ignoring holidays and weekends). Figures 1 and 2 show plots of the data. The models under consideration were discussed in Section 1 (see Table1).

\subsection{Log Volatility Models}

Parameter estimates and log likelihoods for the log volatility models are shown in Table 2 All models are estimated fixing $\eta_{1}^{*}=0$ and with no jump risk premium. Alternative risk premium specifications are discussed in Section 3.3

Including jumps in returns adds nearly 150 points to the log likelihood relative to the model without jumps, consistent with previous findings that jumps provide a big improvement in model fit (e.g., Bates, 2000: Pan, 2002). Whether jumps are scaled by the volatility state $(\operatorname{LogJ} 1)$ or not $(\log J 0)$ makes little difference in log likelihood. But in either case estimated jump distributions differ substantially from what is typically found in the existing literature. We find that jumps occur nearly every other day on average and have positive mean. This issue is discussed in more detail in Section 3.2 
$\log$ SPX
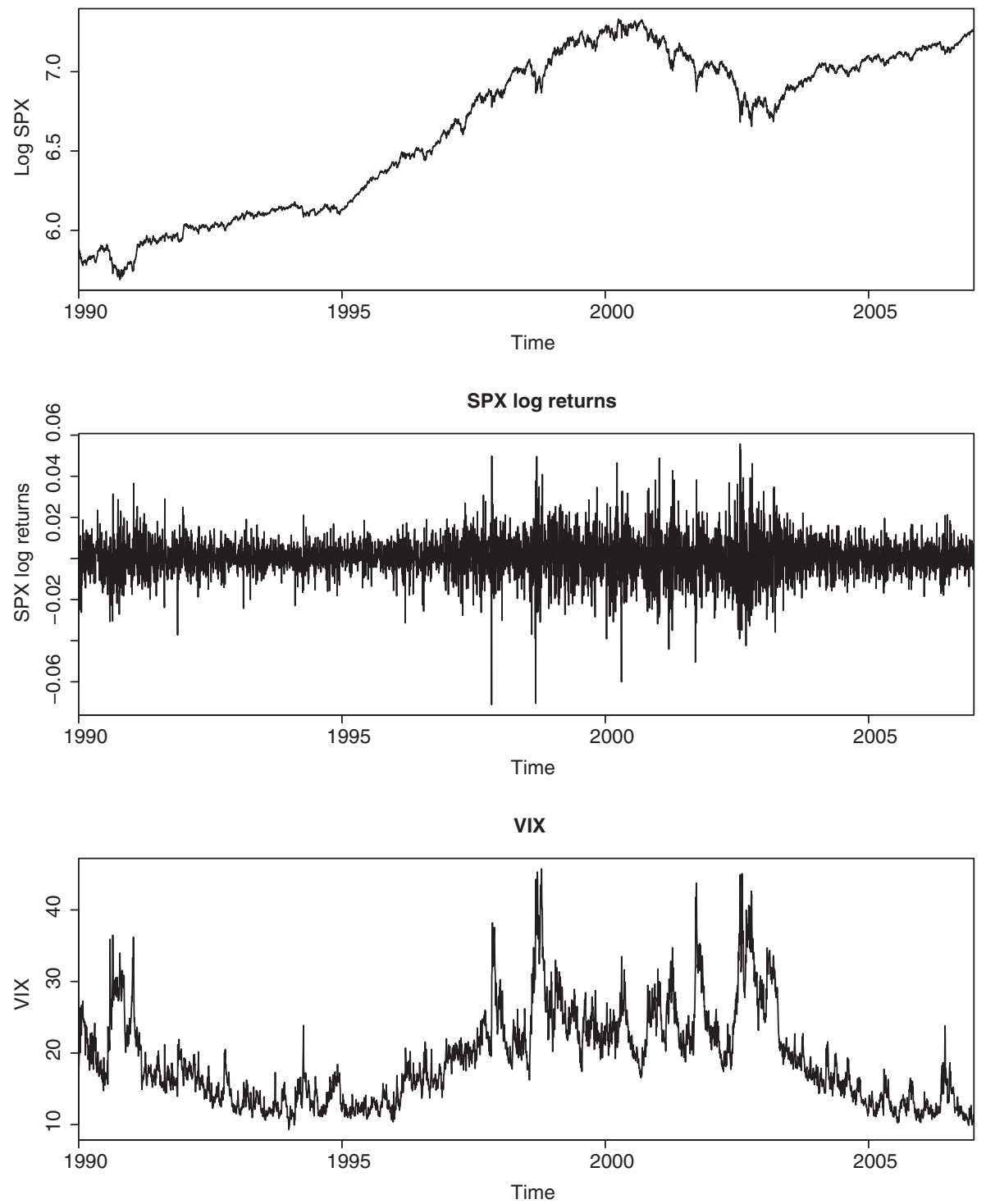

Figure 1 Time-series plots of the log SPX, SPX log returns, and VIX index, January 2, 1990 to December 29, 2006.

Including jumps in volatility (in addition to jumps in returns) provides an additional large gain in log likelihood, consistent with the findings of Eraker,Johannes, and Polson 2003). The best of these models is LogJJ1, which uses scaled and correlated jumps. For this model the improvement in log likelihood 


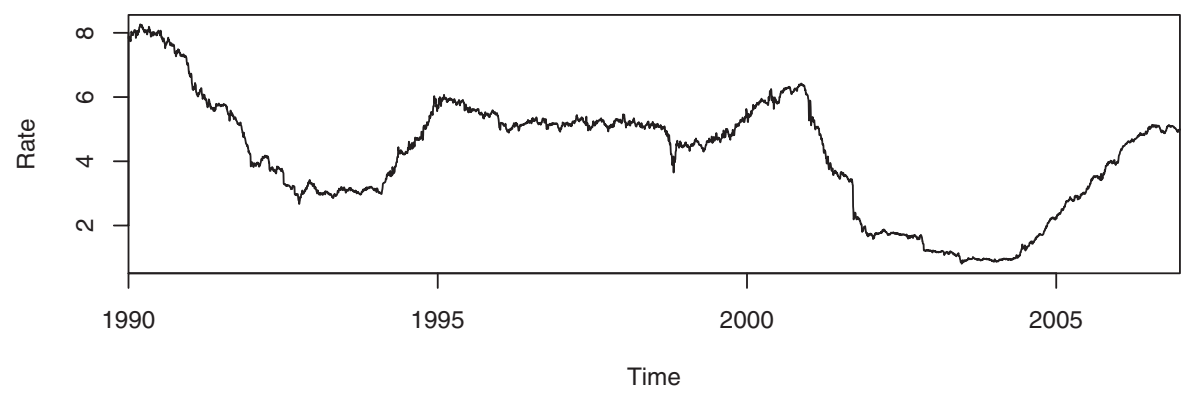

SP 500 dividend rate

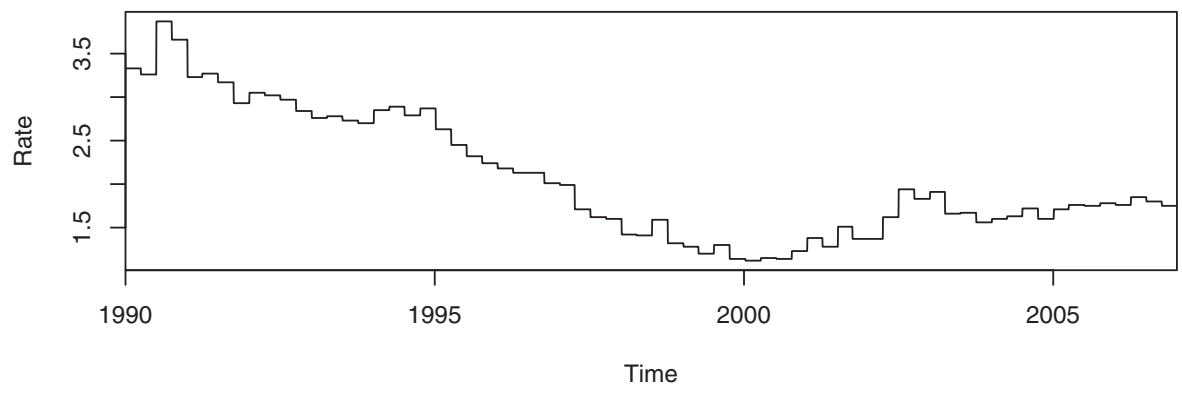

Figure 2 Time-series plots of the three-month Treasury bill rate and S\&P 500 dividend payout rate, January 2, 1990 to December 29, 2006.

is nearly 200 points relative to the models with jumps in returns alone. Jumps occur about every other day on average. The mean of return jumps does not differ significantly from zero (see further discussion on this point in Section 3.2.

Figures 378 show diagnostic plots for the return and volatility generalized residuals discussed in Section 2.3 The figures show results for the Log0, LogJ1, and LogJJ1 models (no jumps, jumps in returns only and jumps in both returns and volatility, respectively).

Figure 3 shows normal-quantile plots for the return residuals. Log0 shows the expected problems in the left tail. The model is unable to account for days with large negative returns. LogJ1 does a little better, but the problem still exists. The issue is largely (but not entirely) resolved by LogJJ1.

Figure 4 shows normal-quantile plots for the volatility residuals. Again, $\log 0$ and $\log 11$, which do not include jumps in volatility, fail badly. The issue is most severe in the right tail, but there are problems in the left tail as well. LogJJ1 does much better, but some unexplained tail fatness remains, especially in the right tail.

Better results might be obtainable with more flexible distributions, e.g., additional jump processes. See also Durham 2007) for an alternative approach using mixtures of normals. 
Table 2 Parameter estimates and log likelihoods for log volatility models

\begin{tabular}{|c|c|c|c|c|c|c|c|}
\hline & $\log$ & LogJ0 & LogJ1 & LogJJ0 & LogJJ1 & $\operatorname{LogJJ} 2$ & LogJJ3 \\
\hline$\alpha_{0} \cdot 10^{3}$ & $\begin{array}{c}0.492 \\
(0.162)\end{array}$ & $\begin{array}{c}0.227 \\
(0.159)\end{array}$ & $\begin{array}{c}0.217 \\
(0.174)\end{array}$ & $\begin{array}{c}0.211 \\
(0.157)\end{array}$ & $\begin{array}{c}0.415 \\
(0.168)\end{array}$ & $\begin{array}{c}0.183 \\
(0.157)\end{array}$ & $\begin{array}{c}0.273 \\
(0.176)\end{array}$ \\
\hline$\alpha_{1}$ & $\begin{array}{c}-3.191 \\
(2.175)\end{array}$ & $\begin{array}{c}0.559 \\
(2.221)\end{array}$ & $\begin{array}{c}0.925 \\
(2.895)\end{array}$ & $\begin{array}{c}0.761 \\
(2.368)\end{array}$ & $\begin{array}{c}-3.494 \\
(3.629)\end{array}$ & $\begin{array}{c}1.754 \\
(2.276)\end{array}$ & $\begin{array}{c}-0.200 \\
(2.905)\end{array}$ \\
\hline$\kappa$ & $\begin{array}{r}-0.0075 \\
(0.0016)\end{array}$ & $\begin{array}{c}-0.0137 \\
(0.0018)\end{array}$ & $\begin{array}{c}-0.0091 \\
(0.0019)\end{array}$ & $\begin{array}{r}-0.0103 \\
(0.0016)\end{array}$ & $\begin{array}{r}-0.0069 \\
(0.0017)\end{array}$ & $\begin{array}{c}-0.0150 \\
(0.0018)\end{array}$ & $\begin{array}{r}-0.0078 \\
(0.0017)\end{array}$ \\
\hline$\mu_{V}$ & $\begin{array}{r}-9.589 \\
(0.259)\end{array}$ & $\begin{array}{r}-9.847 \\
(0.174)\end{array}$ & $\begin{array}{r}-9.837 \\
(0.221)\end{array}$ & $\begin{array}{r}-9.885 \\
(0.211)\end{array}$ & $\begin{array}{r}-10.088 \\
(0.282)\end{array}$ & $\begin{array}{r}-9.877 \\
(0.153)\end{array}$ & $\begin{array}{r}-9.823 \\
(0.240)\end{array}$ \\
\hline$\sigma_{V}$ & $\begin{array}{c}0.122 \\
(0.002)\end{array}$ & $\begin{array}{c}0.151 \\
(0.004)\end{array}$ & $\begin{array}{c}0.125 \\
(0.003)\end{array}$ & $\begin{array}{c}0.113 \\
(0.003)\end{array}$ & $\begin{array}{c}0.087 \\
(0.003)\end{array}$ & $\begin{array}{c}0.135 \\
(0.004)\end{array}$ & $\begin{array}{c}0.109 \\
(0.003)\end{array}$ \\
\hline$\rho$ & $\begin{array}{r}-0.680 \\
0.006\end{array}$ & $\begin{array}{r}-0.795 \\
0.009\end{array}$ & $\begin{array}{c}-0.804 \\
(0.013)\end{array}$ & $\begin{array}{c}-0.757 \\
(0.011)\end{array}$ & $\begin{array}{c}-0.732 \\
(0.013)\end{array}$ & $\begin{array}{c}-0.851 \\
(0.011)\end{array}$ & $\begin{array}{r}-0.826 \\
(0.014)\end{array}$ \\
\hline $\log \left(\lambda_{1}\right)$ & & $\begin{array}{c}-0.672 \\
(0.146)\end{array}$ & $\begin{array}{c}-0.935 \\
(0.236)\end{array}$ & $\begin{array}{c}-1.198 \\
(0.128)\end{array}$ & $\begin{array}{c}-0.893 \\
(0.142)\end{array}$ & $\begin{array}{c}-0.163 \\
(0.163)\end{array}$ & $\begin{array}{c}-1.017 \\
(0.321)\end{array}$ \\
\hline$\mu_{1}$ & & $\begin{array}{l}0.00161 \\
0.00029\end{array}$ & $\begin{array}{c}0.300 \\
(0.057)\end{array}$ & $\begin{array}{c}0.00035 \\
(0.00045)\end{array}$ & $\begin{array}{c}0.006 \\
(0.066)\end{array}$ & $\begin{array}{c}0.00137 \\
(0.00025)\end{array}$ & $\begin{array}{c}0.360 \\
(0.084)\end{array}$ \\
\hline$\sigma_{1}$ & & $\begin{array}{c}0.00506 \\
(0.00029)\end{array}$ & $\begin{array}{c}0.876 \\
(0.061)\end{array}$ & $\begin{array}{c}0.00650 \\
(0.00039)\end{array}$ & $\begin{array}{l}1.241 \\
(0.075)\end{array}$ & $\begin{array}{c}0.00387 \\
(0.00027)\end{array}$ & $\begin{array}{c}0.775 \\
(0.070)\end{array}$ \\
\hline $\log \left(\lambda_{2}\right)$ & & & & & & $\begin{array}{c}-1.922 \\
(0.189)\end{array}$ & $\begin{array}{c}-2.074 \\
(0.214)\end{array}$ \\
\hline$\mu_{2}$ & & & & $\begin{array}{c}0.028 \\
(0.007)\end{array}$ & $\begin{array}{c}0.024 \\
(0.006)\end{array}$ & $\begin{array}{c}0.014 \\
(0.010)\end{array}$ & $\begin{array}{c}0.010 \\
(0.009)\end{array}$ \\
\hline$\sigma_{2}$ & & & & $\begin{array}{c}0.148 \\
(0.007)\end{array}$ & $\begin{array}{c}0.127 \\
(0.007)\end{array}$ & $\begin{array}{c}0.175 \\
(0.013)\end{array}$ & $\begin{array}{c}0.142 \\
(0.012)\end{array}$ \\
\hline$\rho_{J}$ & & & & $\begin{array}{c}-0.489 \\
(0.045)\end{array}$ & $\begin{array}{r}-0.630 \\
(0.031)\end{array}$ & & \\
\hline$\eta_{0} \cdot 10^{2}$ & $\begin{array}{c}4.686 \\
(0.224)\end{array}$ & $\begin{array}{c}5.522 \\
(0.293)\end{array}$ & $\begin{array}{c}4.372 \\
(0.251)\end{array}$ & $\begin{array}{c}5.834 \\
(0.319)\end{array}$ & $\begin{array}{c}4.566 \\
(0.299)\end{array}$ & $\begin{array}{c}5.720 \\
(0.301)\end{array}$ & $\begin{array}{c}4.544 \\
(0.238)\end{array}$ \\
\hline $\log \mathrm{L}$ & $41,109.29$ & $41,262.14$ & $41,256.99$ & $41,430.00$ & $41,455.12$ & $41,421.47$ & $41,391.70$ \\
\hline
\end{tabular}

Standard errors in parentheses. SPX and VIX indices, January 2, 1990 to December 29, 2006.

Figures $5 \sqrt{8}$ show autocorrelation plots for return residuals, volatility residuals, squared return residuals, and squared volatility residuals, respectively. There is little difference amongst the models for these diagnostics. All three show mild negative autocorrelation in returns (Figure 5) and stronger negative autocorrelation in volatility (Figure 6 through the first several lags. All three models exhibit mild positive autocorrelation in squared returns (Figure 7). The relatively strong positive autocorrelation in squared volatility residuals (Figure 8) is the most worrisome issue turned up by these diagnostics. The evidence points toward the presence of stochastic volatility of (log) volatility and the need for a second volatility factor. 


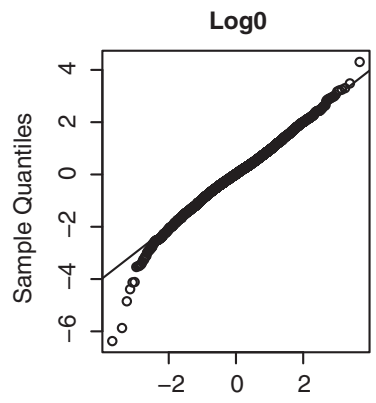

Theoretical Quantiles

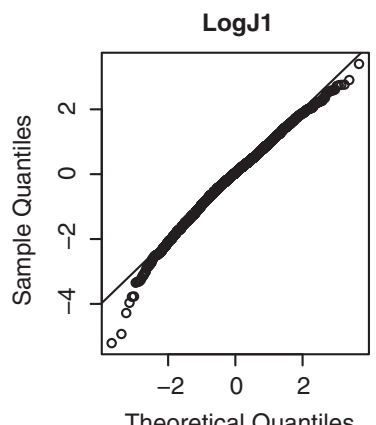

Theoretical Quantiles

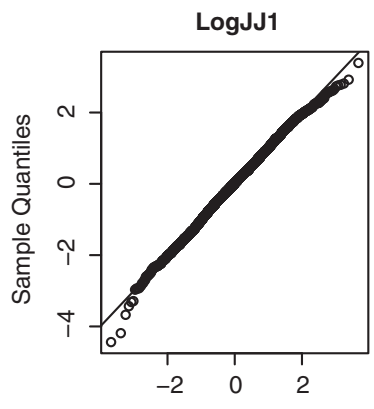

Theoretical Quantiles

Figure 3 Normal-quantile plots for generalized return residuals.
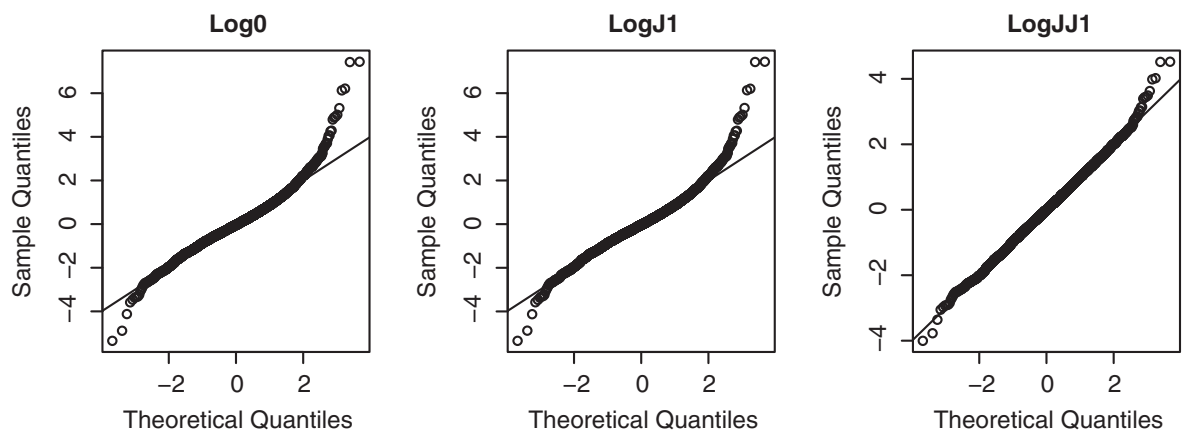

Figure 4 Normal-quantile plots for generalized volatility residuals.
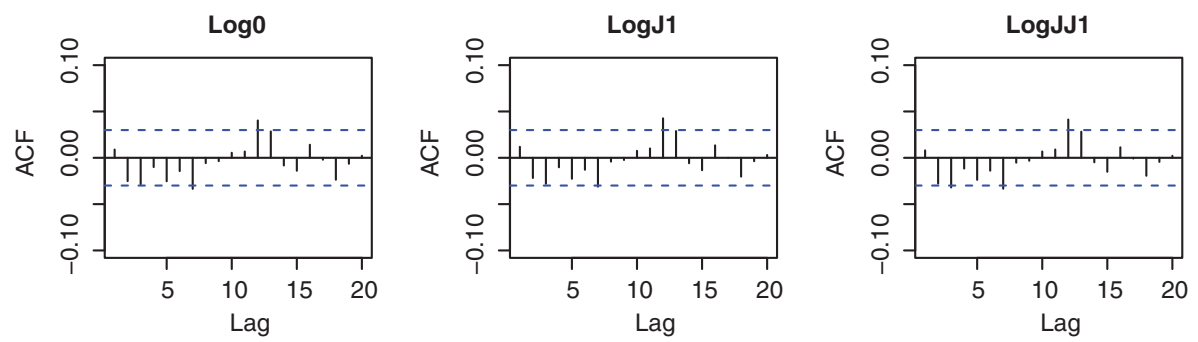

Figure 5 Autocorrelation plots for generalized return residuals.

\subsection{Discussion of Jump Distributions}

The estimated jump distributions found in this article differ substantially from much of the previous literature. While we find that jumps in returns are frequent (around one every other day on average) and have near zero or even positive mean, the existing literature has typically found jumps in returns to occur relatively rarely 

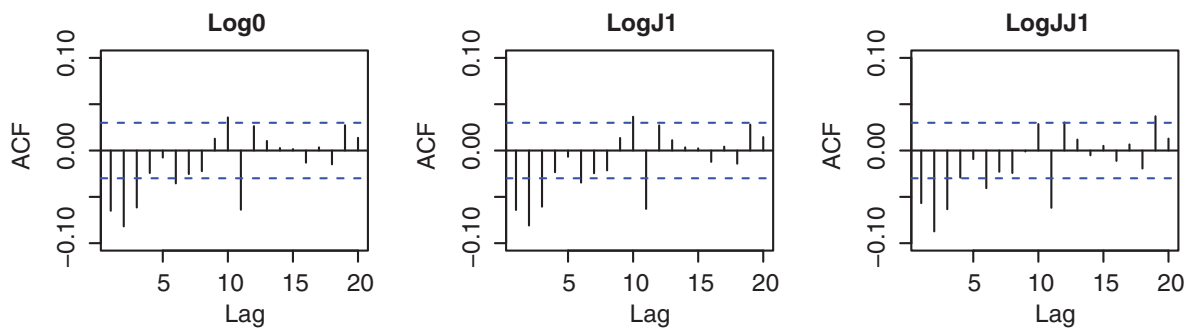

Figure 6 Autocorrelation plots for generalized volatility residuals.
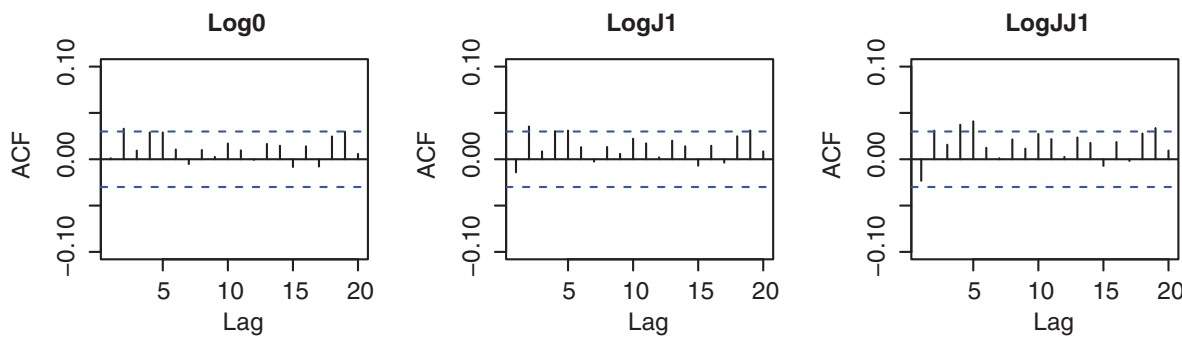

Figure 7 Autocorrelation plots for squared generalized return residuals.
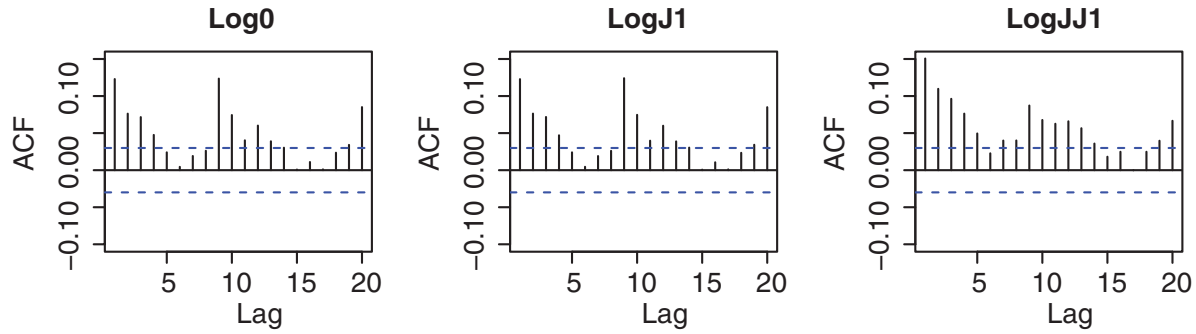

Figure 8 Autocorrelation plots for squared generalized volatility residuals.

and have large, negative mean (e.g., Pan, 2002). The form of the jump distributions typically found in the previous literature is intuitively appealing given that it corresponds to the "crash" days readily apparent in the data. So in this section we investigate the plausibility of our findings. We defer a more detailed discussion of previous work to Section 3.5 following the presentation of our results for affine models. However, we note that Ferriani and Pastorello 2011), who use models and techniques similar to those used in this article, find return jump distributions that are consistent with those reported here. 
Log $\mathbf{1}$

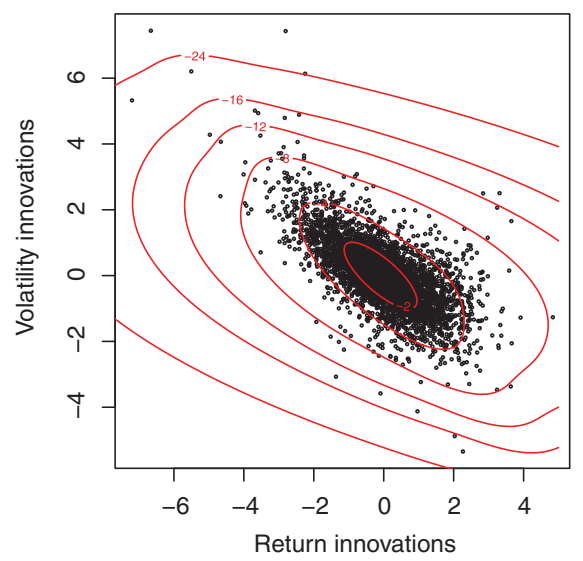

LogJJ1

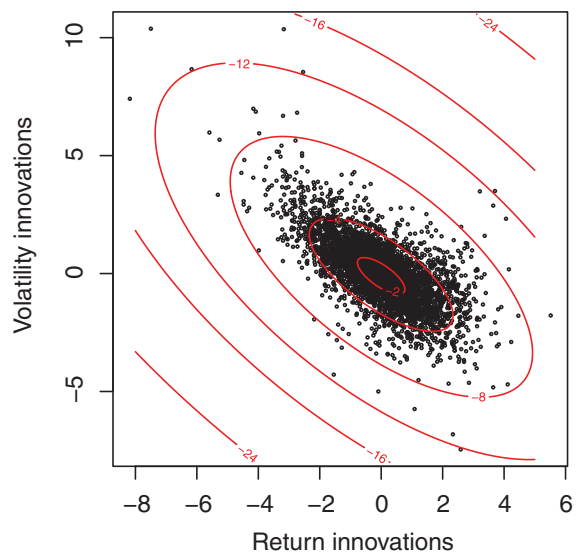

Figure 9 Model innovations, calculated as in 4 with contours of the associated log densities, calculated as in 5 .

Figure 9 shows model innovations calculated as in (4) for LogJ1 and LogJJ1 with parameters reported in Table 2 Also shown are contours of the associated log densities, calculated as in (5).

Visual inspection of this figure suggests that the estimated densities found by the optimizer are reasonable, although more flexible models (e.g., multiple jump components) could potentially provide somewhat better fits. The most severe problems that the models need to address are with respect to the volatility innovations, so they expend most of their available degrees of freedom trying to fit that feature of the data. These figures and the normal-quantile plots in the previous subsection suggest that the models do about as well as might be hoped given the limited range of flexibility available to them.

But some practitioners may feel strongly about the existence of infrequent return jumps with large negative mean. As an experiment, we tried refitting the LogJ1 and LogJJ1 models with jump parameters fixed at various settings designed to reflect such beliefs. In each case, all other model parameters were estimated conditional on the fixed jump parameters.

For example, Figure 10 shows model innovations and density contours analogous to Figure 9 but with jump parameters fixed at $\lambda_{1}=0.002, \mu_{1}=-2.0$, and $\sigma_{1}=2.0$ (these parameter values are consistent with results from the extant literature summarized in Table I of Broadie, Chernov, and Johannes 2007); experiments with alternative parameter settings gave similar results). The results for LogJJ1 look reasonable. It does a little better at capturing the several observations in the upper left corner of the figure. However, it does a little worse in other parts of the distribution where there are far more observations. The log likelihood for the 
Log $\mathbf{1}$

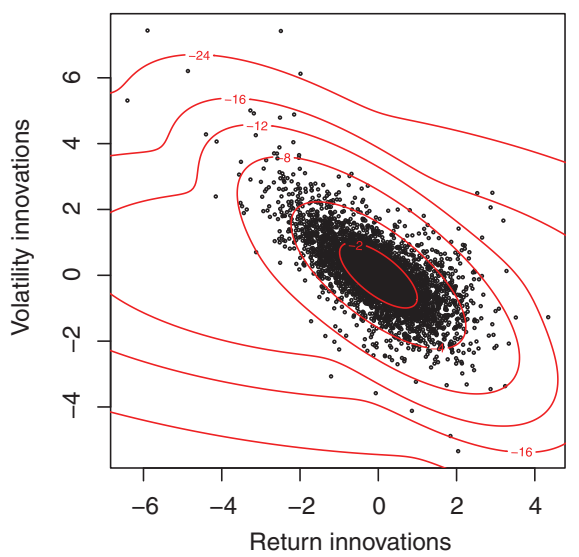

LogJJ1

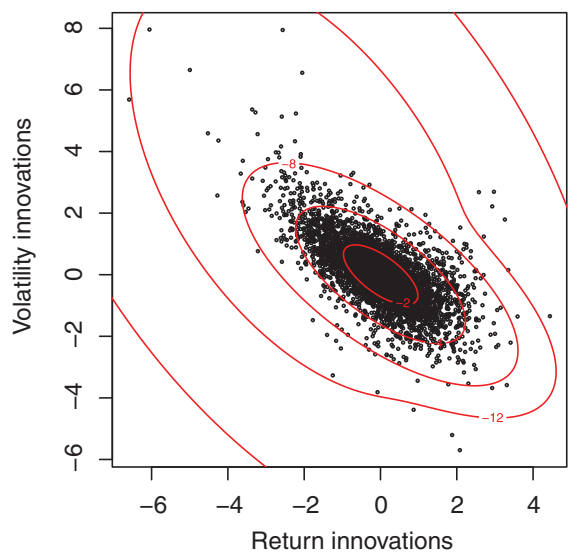

Figure 10 Model innovations, analogous to Figure 9 but with return jump parameters fixed at $\lambda_{1}=0.002, \mu_{1}=-2.0$, and $\sigma_{1}=2.0$. All other model parameters are fully optimized conditional on these fixed jump parameters.

constrained model is about 170 points worse than for the unconstrained model with parameter values shown in Table_ The results for LogJ1 are more interesting. Including an extreme left-tail jump component in returns when there are no jumps in volatility simply does not appear to be useful. While naive intuition may suggest that such distributions could be plausible, they put significant probability mass where there are few observations while failing to put additional mass in the region where the targeted extreme observations are actually located.

Ultimately, we are interested in the shape of the predictive densities implied by a particular model, not so much in the parameters themselves. None of these models represent the true data-generating process. We do not argue that our estimates of the jump process are correct and the estimates found in previous work incorrect. They are simply different ways of trying to fit misspecified models. Nonetheless, optimizers are quite good at optimizing, and the maximum likelihood estimator does have the attribute of minimizing Kullback-Leibler distance.

The practitioner with strong prior beliefs regarding the existence of infrequent jumps with large negative mean can impose them on the model. But the loss in model fit is substantial.

\subsection{Discussion of Risk Premia}

As described in Section 2.1, the risk-neutral model implies a mapping from latent volatility states to corresponding values of IV, a model-free measure of volatility based on observed option prices. Volatility dynamics implied by different 
(a)

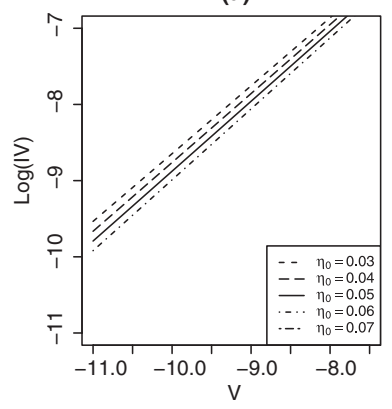

(b)

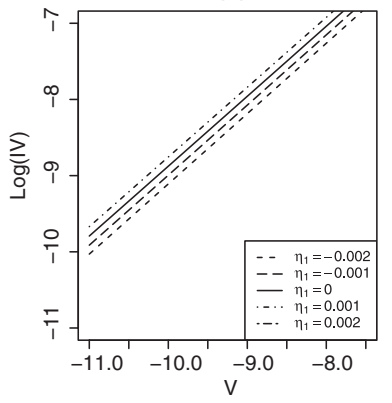

(c)

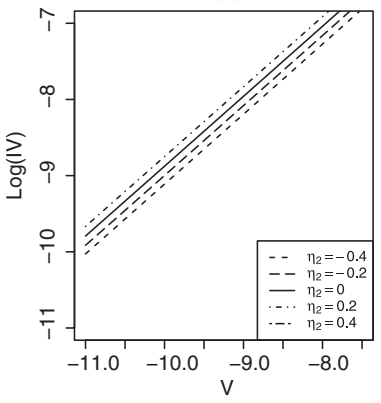

Figure 11 Mappings from implied to observed states for alternative settings of risk-premium parameters, LogJJ1. Panels (a), (b), and (c) show mappings corresponding to various values of $\eta_{0}^{*}$, $\eta_{1}^{*}$, and $\eta_{2}^{*}$, respectively. For each panel, all other model parameters are fixed at the values reported in Table 2

risk-neutral specifications or parameter values generate different mappings. The maximum likelihood estimator optimizes across candidate parameter vectors to find the mapping for which the volatility states corresponding to observed IV provide the best fit to observed returns (conditional on the parametric constraints imposed by a particular model).

In this section, we examine the implications of various assumptions for the diffusive risk premia, $\eta_{0}^{*}$ and $\eta_{1}^{*}$, and a jump risk premium, $\eta_{2}^{*}$. We assume the jump risk premium is such that $\left.\mathrm{E}[]_{1 t}^{* 2}\right]=\exp \left(V_{t}\right)\left(\mu_{1}^{2}+\sigma_{1}^{2}+\eta_{2}^{*}\right)$ for the scaled jump models and $\left.\mathrm{E}[]_{1 t}^{* 2}\right]=\mu_{1}^{2}+\sigma_{1}^{2}+\eta_{2}^{*}$ for the unscaled models (note that the only place the jump distribution under the risk-neutral measure enters the likelihood is through the second moment of jump size in Equation (2)).

Figure 11 shows mappings from volatility state to IV for the LogJJ1 model corresponding to several alternative values for $\eta_{0}^{*}$ (left panel), $\eta_{1}^{*}$ (center panel), and $\eta_{2}^{*}$ (right panel). In each panel, all other parameters are held fixed at the values shown in Table2

There is little difference among the shapes of the mappings that result from varying the different risk premium parameters. In each case, there is an upward shift from volatility state to log IV, with the magnitude of this shift determined by the size of the risk premium. But it makes little difference which of $\eta_{0}^{*}, \eta_{1}^{*}$, or $\eta_{2}^{*}$ is varied. All have essentially the same effect, making identification difficult. If the model is fully optimized with any combination of these estimated as free parameters, the log likelihood and other model parameters differ only minimally from values reported in Table 2

In the context of Pastorello, Patilea, and Renaul 2003), $\eta^{*}=\left(\eta_{0}^{*}, \eta_{1}^{*}, \eta_{2}^{*}\right)$ can be thought of as a vector of nuisance parameters that determines (in conjunction with the other model parameters) the mapping from observed to implied states. This parameter vector is poorly identified. That is, different choices for $\eta^{*}$ can 
Table 3 Parameter estimates and log likelihoods for affine models

\begin{tabular}{|c|c|c|c|c|c|c|c|}
\hline & Aff & AffJ0 & AffJ1 & AffJJ0 & AffJJ1 & AffJJ2 & AffJJ3 \\
\hline$\alpha_{0} \cdot 10^{3}$ & $\begin{array}{c}-0.011 \\
(0.120)\end{array}$ & $\begin{array}{c}0.322 \\
(0.184)\end{array}$ & $\begin{array}{c}-0.004 \\
(0.194)\end{array}$ & $\begin{array}{c}-0.603 \\
(0.198)\end{array}$ & $\begin{array}{c}-0.151 \\
(0.179)\end{array}$ & $\begin{array}{c}0.282 \\
(0.173)\end{array}$ & $\begin{array}{c}-0.002 \\
(0.180)\end{array}$ \\
\hline$\alpha_{1}$ & $\begin{array}{c}3.023 \\
(2.256)\end{array}$ & $\begin{array}{c}-0.495 \\
(2.733)\end{array}$ & $\begin{array}{c}4.242 \\
(3.012)\end{array}$ & $\begin{array}{l}10.554 \\
(2.977)\end{array}$ & $\begin{array}{c}6.456 \\
(2.907)\end{array}$ & $\begin{array}{c}0.008 \\
(2.728)\end{array}$ & $\begin{array}{c}4.286 \\
(3.025)\end{array}$ \\
\hline$\kappa$ & $\begin{array}{c}-0.016 \\
(0.002)\end{array}$ & $\begin{array}{c}-0.012 \\
(0.003)\end{array}$ & $\begin{array}{c}-0.012 \\
(0.003)\end{array}$ & $\begin{array}{c}-0.038 \\
(0.003)\end{array}$ & $\begin{array}{c}-0.034 \\
(0.003)\end{array}$ & $\begin{array}{c}-0.023 \\
(0.003)\end{array}$ & $\begin{array}{c}-0.024 \\
(0.003)\end{array}$ \\
\hline$\mu_{V} \cdot 10^{3}$ & $\begin{array}{c}0.097 \\
(0.011)\end{array}$ & $\begin{array}{c}0.077 \\
(0.017)\end{array}$ & $\begin{array}{c}0.082 \\
(0.016)\end{array}$ & $\begin{array}{c}0.084 \\
(0.006)\end{array}$ & $\begin{array}{c}0.086 \\
(0.006)\end{array}$ & $\begin{array}{c}0.077 \\
(0.008)\end{array}$ & $\begin{array}{c}0.081 \\
(0.008)\end{array}$ \\
\hline$\sigma_{V} \cdot 10^{3}$ & $\begin{array}{c}1.380 \\
(0.024)\end{array}$ & $\begin{array}{c}1.371 \\
(0.027)\end{array}$ & $\begin{array}{c}1.324 \\
(0.029)\end{array}$ & $\begin{array}{c}1.023 \\
(0.028)\end{array}$ & $\begin{array}{c}1.059 \\
(0.028)\end{array}$ & $\begin{array}{c}1.218 \\
(0.031)\end{array}$ & $\begin{array}{c}1.142 \\
(0.031)\end{array}$ \\
\hline$\rho$ & $\begin{array}{c}-0.658 \\
(0.007)\end{array}$ & $\begin{array}{c}-0.771 \\
(0.010)\end{array}$ & $\begin{array}{c}-0.786 \\
(0.012)\end{array}$ & $\begin{array}{r}-0.663 \\
(0.010)\end{array}$ & $\begin{array}{c}-0.671 \\
(0.010)\end{array}$ & $\begin{array}{c}-0.781 \\
(0.010)\end{array}$ & $\begin{array}{r}-0.768 \\
(0.013)\end{array}$ \\
\hline $\log \left(\lambda_{1}\right)$ & & $\begin{array}{c}-0.321 \\
(0.137)\end{array}$ & $\begin{array}{c}-1.115 \\
(0.223)\end{array}$ & $\begin{array}{r}-2.490 \\
(0.116)\end{array}$ & $\begin{array}{c}-2.860 \\
(0.133)\end{array}$ & $\begin{array}{r}-0.198 \\
(0.167)\end{array}$ & $\begin{array}{r}-1.367 \\
(0.264)\end{array}$ \\
\hline$\mu_{1}$ & & $\begin{array}{c}0.0014 \\
(0.0002)\end{array}$ & $\begin{array}{c}0.341 \\
(0.063)\end{array}$ & $\begin{array}{c}0.0016 \\
(0.0014)\end{array}$ & $\begin{array}{c}-0.019 \\
(0.208)\end{array}$ & $\begin{array}{c}0.0008 \\
(0.0002)\end{array}$ & $\begin{array}{c}0.230 \\
(0.073)\end{array}$ \\
\hline$\sigma_{1}$ & & $\begin{array}{c}0.0044 \\
(0.0002)\end{array}$ & $\begin{array}{c}0.921 \\
(0.070)\end{array}$ & $\begin{array}{c}0.0087 \\
(0.0008)\end{array}$ & $\begin{array}{c}1.288 \\
(0.104)\end{array}$ & $\begin{array}{c}0.0042 \\
(0.0003)\end{array}$ & $\begin{array}{c}1.023 \\
(0.087)\end{array}$ \\
\hline $\log \left(\lambda_{2}\right)$ & & & & & & $\begin{array}{c}-3.355 \\
(0.191)\end{array}$ & $\begin{array}{r}-3.408 \\
(0.190)\end{array}$ \\
\hline$\mu_{2} \cdot 10^{5}$ & & & & $\begin{array}{c}2.267 \\
(0.203)\end{array}$ & $\begin{array}{c}2.673 \\
(0.292)\end{array}$ & $\begin{array}{c}2.365 \\
(0.341)\end{array}$ & $\begin{array}{c}2.477 \\
(0.355)\end{array}$ \\
\hline$\rho_{J}$ & & & & $\begin{array}{r}-0.0096 \\
(0.0015)\end{array}$ & $\begin{array}{c}-0.739 \\
(0.200)\end{array}$ & & \\
\hline$\eta_{1} \cdot 10^{2}$ & $\begin{array}{c}4.869 \\
(0.237)\end{array}$ & $\begin{array}{c}5.608 \\
(0.336)\end{array}$ & $\begin{array}{r}-11.030 \\
(12.988)\end{array}$ & $\begin{array}{c}8.338 \\
(0.413)\end{array}$ & $\begin{array}{l}24.915 \\
(3.794)\end{array}$ & $\begin{array}{c}6.669 \\
(0.362)\end{array}$ & $\begin{array}{l}10.655 \\
(3.747)\end{array}$ \\
\hline$\eta_{2} \cdot 10^{5}$ & & $\begin{array}{c}0.006 \\
(0.005)\end{array}$ & & $\begin{array}{c}-0.521 \\
(0.086)\end{array}$ & & $\begin{array}{c}-0.007 \\
(0.005)\end{array}$ & \\
\hline$\eta_{2}$ & & & $\begin{array}{c}10.199 \\
(12.294)\end{array}$ & & $\begin{array}{r}-18.369 \\
(2.391)\end{array}$ & & $\begin{array}{c}-2.503 \\
(1.290)\end{array}$ \\
\hline
\end{tabular}

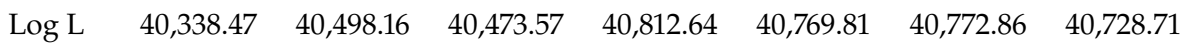

Standard errors in parentheses. SPX and VIX indices, January 2, 1990 to December 29, 2006.

generate essentially the same mapping. But this has no effect on identification of the parameters of the physical model (which depends only on the mapping from observed to implied states, not the particular value of $\eta^{*}$ used to construct it).

\subsection{Affine Models}

Parameter estimates and log likelihoods for the affine models are shown in Table 3 In contrast to the log volatility models, including a jump risk premium does improve 
the performance of the affine models, so the results in Table 3 and elsewhere in this section all include a jump risk premium. The form of the jump risk premium is analogous to the one described in the context of the log volatility models (see Section 3.3. We also estimate $\eta_{1}^{*}$ but fix $\eta_{0}^{*}=0$, following standard practice (e.g., Bates, 2000: see also discussion of alternative risk premium specifications below).

Although we do include a jump risk parameter in the model, our estimates of it may not have much practical value. As noted by Broadie, Chernov, and Johannes 2007), more information (e.g., from either the term structure of implied volatility or shape of the implied volatility smile across moneyness) is needed to disentangle the effects of the various sources of risk premium in any meaningful way. This article makes no use of such information, nor does it intend to have explanatory power for these features of the data.

As with the log volatility models and consistent with the existing literature (e.g., Pan, 2002), including jumps in returns provides a huge increase in log likelihood (about 160 points). In contrast to the log volatility models, the model with unscaled jumps does better here. Including jumps in volatility provides additional large improvements in log likelihood. The best performing model is AffJJ0, which uses unscaled, correlated jumps. This model is about 40 points in log likelihood better than the next best alternative, AffJJ2 (unscaled, uncorrelated jumps), and over 300 points better than the best of the models that does not include jumps in volatility.

Similar issues exist here with respect to the frequency and size distribution of jumps as were manifest with the log volatility models, and we refer the reader back to the discussion in Section 3.2 on this point. A more detailed discussion of the existing literature is deferred to Section 3.5

While including jumps in returns and volatility improves the affine model a great deal, these models are all heavily dominated by the log volatility models. The best of the affine models is over 600 points in log likelihood worse than the best log volatility model. Indeed, the best affine model is nearly 300 points worse than the simplest log volatility model, which includes jumps in neither returns nor volatility.

In contrast to the log volatility models, the affine models (with or without jumps) have explosive volatility dynamics under the risk-neutral measure. But this is more likely an artifact of model misspecification than a meaningful feature of the data. Models with explosive volatility dynamics are essentially useless for forecasting volatility at any time horizon other than the specific horizon at which the model is estimated (corresponding to the one-month horizon of the VIX index in this application).

Figures 12 17 show diagnostic plots for several of the affine models. These diagnostics are largely similar to those for the log volatility models shown in Section 3.1 but there are some important differences. Figure 12 which shows normal-quantile plots for generalized return residuals, suggests that AffJJ0 actually does a little better than the best of the log volatility models in fitting the marginal return distribution. However, the normal-quantile plots for the generalized volatility residuals (Figure 13) are more problematic. Including jumps in volatility (AffJJ0) helps a great deal, as expected, and this model matches the right tail of the 

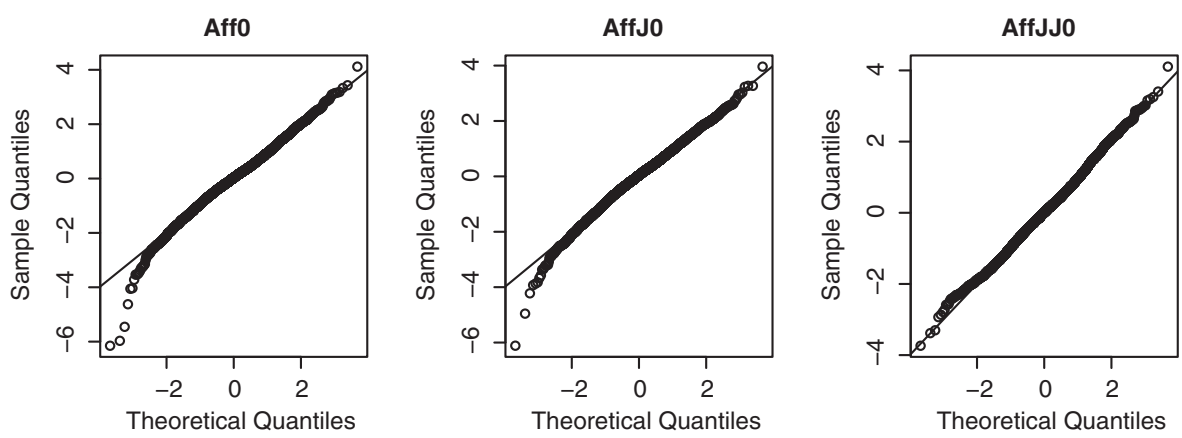

Figure 12 Normal-quantile plots for generalized return residuals.
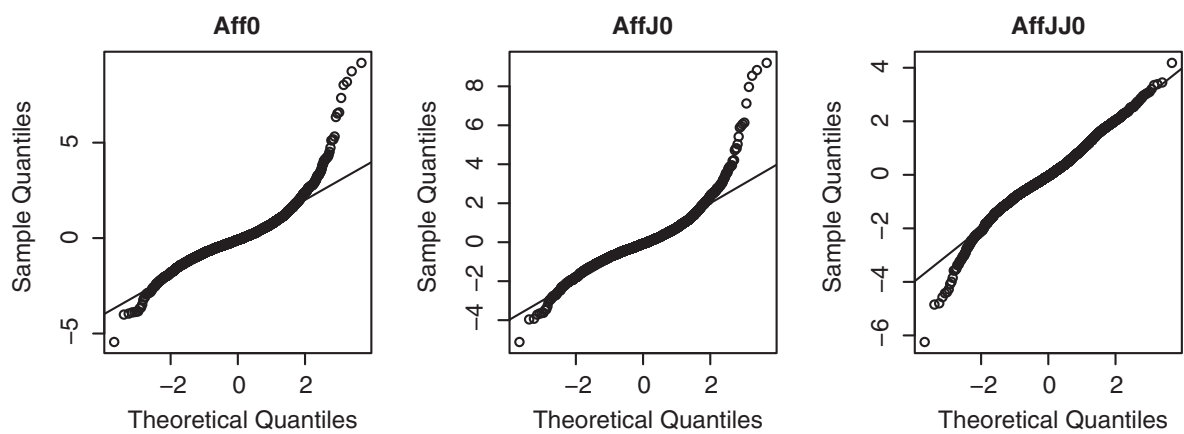

Figure 13 Normal-quantile plots for generalized volatility residuals.
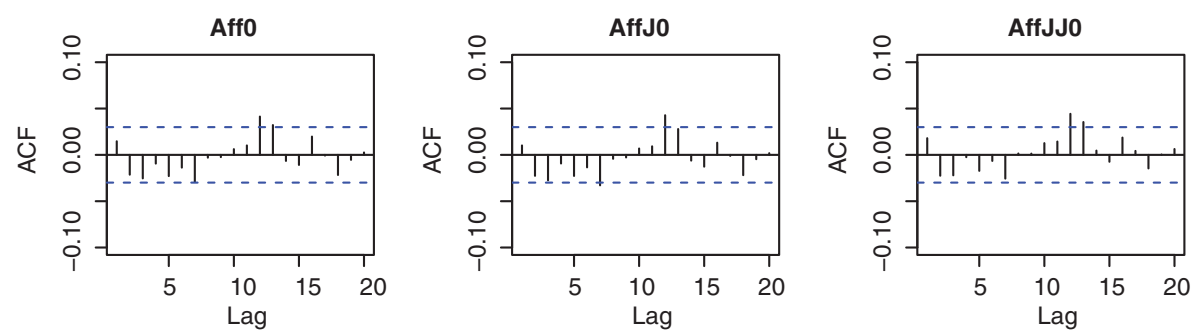

Figure 14 Autocorrelation plots for generalized return residuals.

distribution quite well. But, there are serious problems in the left tail. Given that the exponential distribution, which is used to describe volatility jumps in this model, generates a long tail in one direction (depending on the sign of the coefficient) but nothing in the other, this result should not be a complete surprise.

The autocorrelation plots shown in Figures 14 17 are mostly similar to those for the log models (Section 3.1). However, the autocorrelation plots for squared 

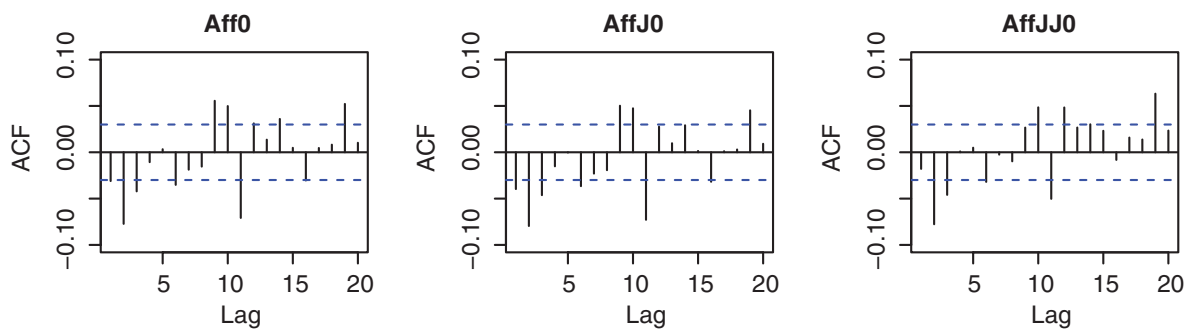

Figure 15 Autocorrelation plots for generalized volatility residuals.
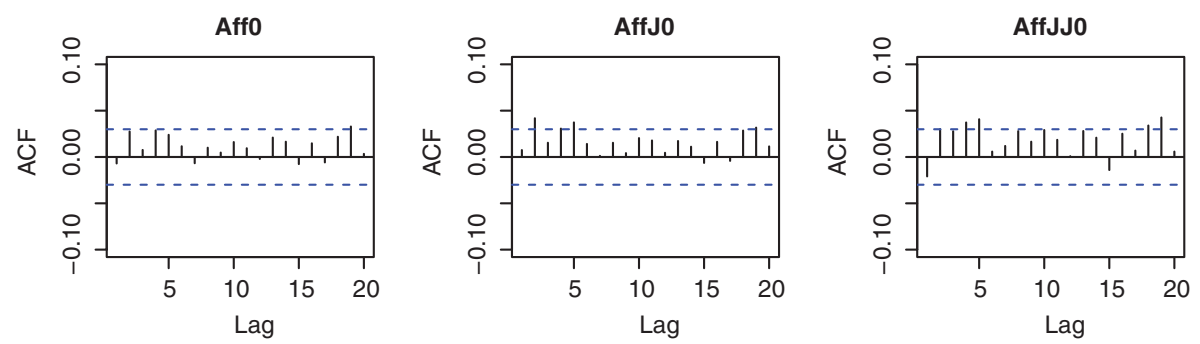

Figure 16 Autocorrelation plots for generalized squared return residuals.
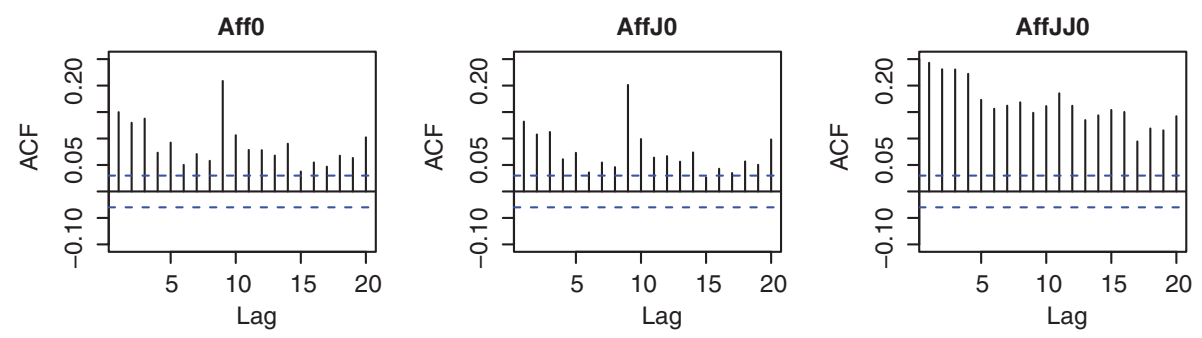

Figure 17 Autocorrelation plots for generalized squared volatility residuals.

volatility residuals (Figure 17) are noticeably worse than those for the log volatility models (Figure 8). The affine models are poorly specified for volatility of volatility, as pointed out by e.g., Poteshman 1998) and Lones 2003).

Figure 18 shows time-series plots of the generalized volatility residuals for AffJ0 and AffJJ0. The autocorrelation pattern is clearly evident in both, although in AffJ0 it is to some extent masked by the extreme outliers (recall that under the hypothesis that the model is the true data-generating process, these should be iid 


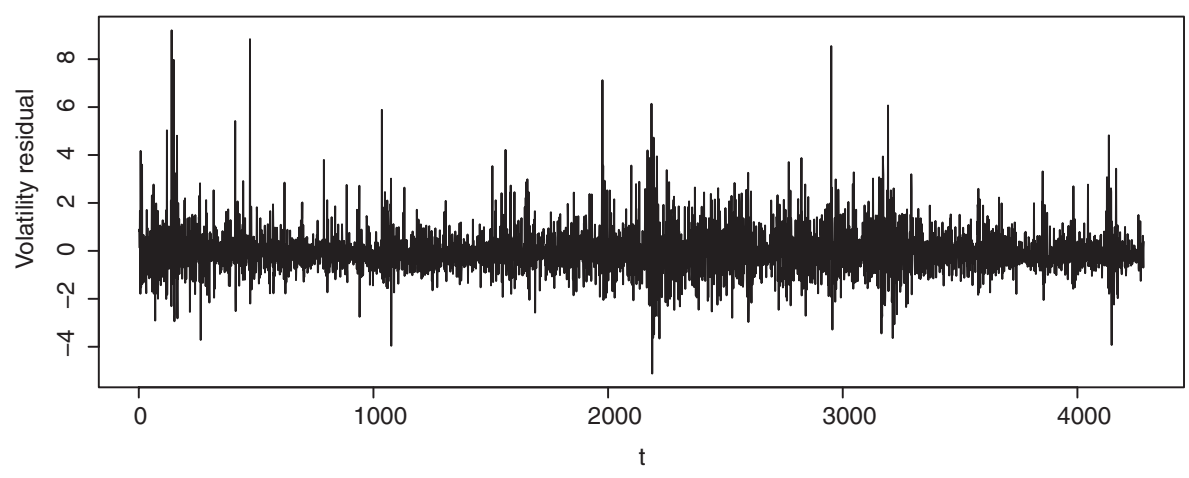

AffJJo

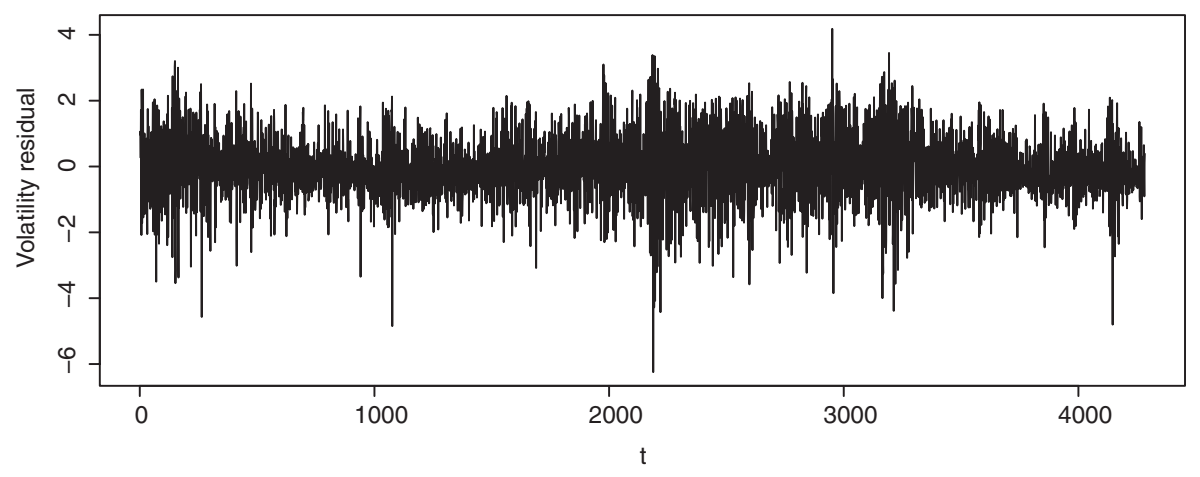

LogJJ0

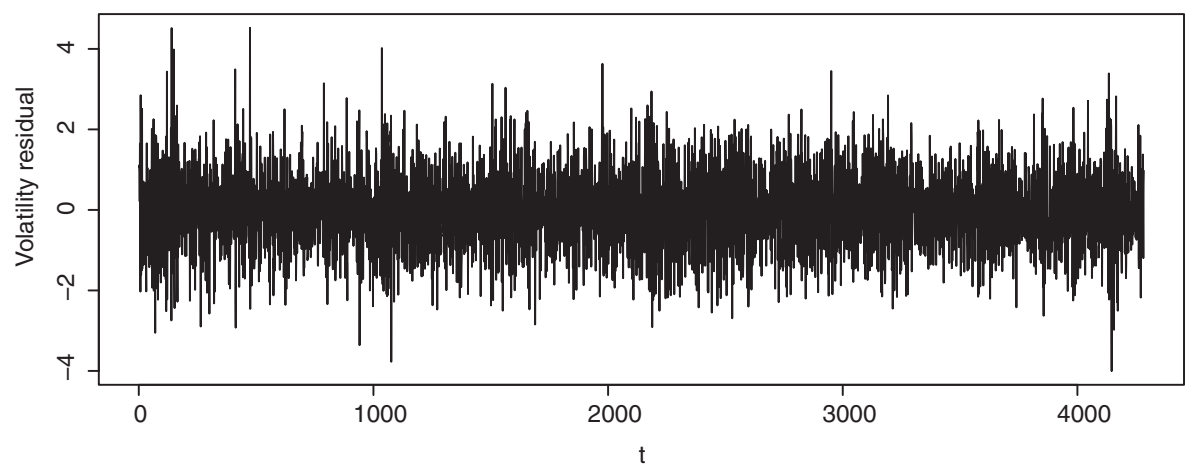

Figure 18 Time series plots of generalized volatility residuals. 
(a)

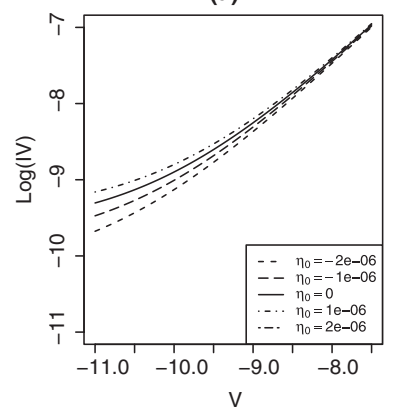

(b)

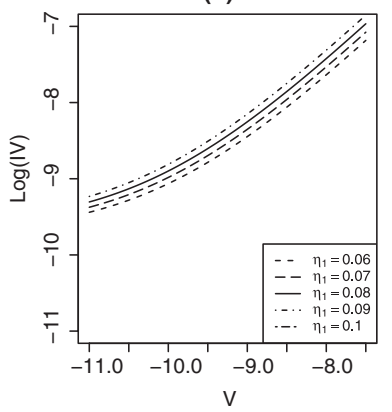

(c)

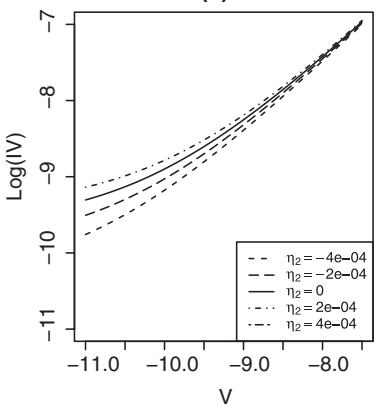

Figure 19 Mappings from implied to observed states for alternative settings of risk-premium parameters, AffJJ0. Panels (a), (b), and (c) show mappings corresponding to various values of $\eta_{0}^{*}$, $\eta_{1}^{*}$, and $\eta_{2}^{*}$, respectively. For each panel, all other model parameters are fixed at the values reported in Table 3

standard normal). In either case, the generalized residuals tend to be too small (in absolute value) when the observed IV is low and too large when the observed IV is high. It makes little difference whether we use scaled or unscaled, correlated or uncorrelated jumps: the resulting figures are nearly identical in all cases. For reference, an analogous plot is shown for LogJJ0. There is still some autocorrelation in the generalized residuals here, but the problem is much less severe. The squareroot specification for volatility of volatility simply does not fit the data. Jumps in volatility help but do not resolve the problem.

Figure 18 also provides an interesting look at the shortcomings of the exponential distribution for jumps in volatility. Recall that generalized residuals that are large in absolute value indicate large innovations that the model has difficulty explaining. Comparison of the upper panel (AffJ0) and middle panel (AffJJ0) shows clearly how including jumps in volatility does a good job at capturing large positive moves in volatility but does essentially nothing with respect to the large negative moves that are also observed.

Figure 19 explores alternative settings for the available risk premium parameters, analogous to Figure 11 in Section 3.3 The figure shows mappings from volatility state to IV corresponding to several alternative values for $\eta_{i}^{*}(i=0,1,2)$. Varying either $\eta_{0}^{*}$ (left panel) or $\eta_{2}^{*}$ (right panel) has essentially the same effect, while the effect of varying $\eta_{1}^{*}$ (center panel) is slightly different. The results shown in Table 3 include both $\eta_{1}^{*}$ and $\eta_{2}^{*}$ as free parameters. Omitting either of these has a significant cost in $\log$ likelihood. Including $\eta_{0}^{*}$ in the model has negligible benefit.

\subsection{Discussion of Previous Work}

This section provides a brief discussion of some findings of prior work regarding jumps and return distributions. 
A number of papers, including Chernov et al. 2003) and Durham 2007, have estimated models based on returns alone and found evidence of long left tails in the return distributions. But, such studies do not make use of the more informative signal about the volatility state that is available using option prices. As noted by Andersen et al. 2001) in the context of high-frequency estimates of the volatility state, if returns are normalized by more informative estimates of volatility, much of this non-Gaussianity may disappear.

Bates 2000) looks at affine models with one or two volatility factors and jumps in returns only. While he finds evidence of infrequent jumps with large negative mean, his findings pertain to risk-neutral rather than physical models.

Pan 2002) looks at affine models that include jumps in returns but not volatility. Under her preferred SVJ0 model, she finds evidence of jumps that are relatively rare with small but negative mean and high dispersion under the physical model. But these findings are difficult to compare to those of this article: she looks at weekly data, which have limited explanatory power regarding the distribution of daily returns; her sample is small (only 8 years of weekly data, about 400 observations); and she uses a simulated method of moments estimator (which can give very different results from likelihood-based estimators in the presence of model misspecification). Although Pan reports that her model is not rejected by the joint time-series of returns and implied volatility states, the results reported in Section 3.4 of this article suggest that this may be because her sample size is small and her tests lack power.

Eraker, Johannes, and Polson 2003) look at affine models with jumps in volatility and returns, including models that are essentially identical to the AffJJ0 and AffJJ2 models examined in this article. They use (likelihood-based) Bayesian methods and find evidence of infrequent return jumps with large negative mean in the physical model. But they rely on returns data alone to extract volatility states. As noted above, this results in a substantially less informative signal about volatility than using option prices. Furthermore, they limit the maximum number of jumps per day to one, which may bias the results in favor of low jump intensity. And finally, their findings may be dictated by the priors they use. They use priors that "are always consistent with the intuition that jumps are 'large' and infrequent." The prior places "low probability on the jump sizes being small" and "low probability on the daily jump probability being greater than 10 percent."

Eraken 2004) looks at affine models with jumps in returns and volatility. Using observed option prices to back out volatility states, Eraker also finds evidence of infrequent jumps with large negative mean. But, as with Eraker, Johannes, and Polson 2003), the maximum number of jumps per day is limited to one. Although Eraker uses Bayesian estimation, his priors are not reported. If they are consistent with Eraker, Johannes, and Polson 2003), however, his results could be biased as noted above. Also, Eraker's sample is relatively small, consisting only of data from January 1, 1987 through December 31, 1990 (about 1000 observations). As noted by Eraker, this sample period may not be representative. 
Broadie, Chernov, and Johannes 2007 look at affine models that include jumps in returns and volatility, but they rely on estimates for the physical model from Eraker, Iohannes, and Polson 2003).

In contrast to the work summarized above, Ferriani and Pastorello 2011) look at log models similar to some of those used in this article (but with jumps in returns only), use a large sample of daily data (January 4, 1996 to December 30, 2005), and apply techniques closely related to those used in this article. In their Log-Ja model (which corresponds to the LogJ0 model in this article), they find that under the physical measure jumps occur frequently, have low dispersion, and have mean near zero. Their findings in this regard are consistent with those of this article.

\section{CONCLUSIONS}

This article demonstrates techniques for joint analysis of physical and risk-neutral models for financial assets. In contrast to much of the existing literature that focuses on affine models for reasons of computational tractability, these techniques are applicable to a broad class of diffusion models, including log volatility models with jumps in both returns and volatility. We demonstrate efficient techniques for inverting the risk-neutral measure in order to get implied volatility states from observed panels of option prices, maximum likelihood estimation, and a highly informative set of diagnostics.

The application looks at SPX and VIX index data. Consistent with previous work, including jumps in returns provides a large increase in log likelihood relative to models with no jumps, and including jumps in volatility provides an additional large increase. In contrast to previous work, we find that return jumps occur frequently, are mostly small, and have near zero mean.

Log volatility models are dramatically better than the corresponding affine models. Including jumps, whether in returns alone or together with jumps in volatility, does not change this result. The best log volatility model is over 600 points in log likelihood better than any of the affine models. The best of the affine models is nearly 300 points worse than even the simple log volatility model with no jumps.

The affine models also exhibit severe problems with the diagnostics. For example, all of the affine models exhibit substantial autocorrelation in squared volatility generalized residuals. The square root specification for volatility of volatility simply does not fit the data; including jumps is of little help here. Also, while affine models with exponential jumps in volatility are able to match the right tail of volatility innovations, they are not able to match the left tail. Exponential jumps are either always positive or always negative, depending on the sign of the coefficient. The fitted models can generate large upward moves in volatility, but not large downward moves, such as are also exhibited by the data.

The availability of powerful diagnostics based on generalized residuals is a useful tool for model exploration. Although it is easy to perform conventional 
tests (e.g., Jarque-Bera or Box-Pierce) using the generalized residuals, we do not report these in the article. All the models are rejected at far beyond conventional significance levels on at least one test. One would have to be almost hopelessly optimistic to believe that any of the models examined in this article was the true data-generating process. Failure to reject a model in exercises such as this should more likely be interpreted as a sign of insufficient sample size or tests with low power rather than an indication that one has found the true data-generating process. Powerful diagnostics are a good thing; failure to find evidence of defective models is a serious liability.

Received August 27, 2012; revised February 7, 2013; accepted February 8, 2013.

\section{REFERENCES}

Andersen, T., T. Bollerslev, F. Diebold, and H. Ebens. 2001. The Distribution of Realized Stock Return Volatility. Journal of Financial Economics 61: 43-76.

Andersen, T., T. Bollerslev, F. Diebold, and P. Labys. 2003. Modeling and Forecasting Realized Volatility. Econometrica 71: 579-625.

Andersen, T. G., L. Benzoni, and J. Lund. 2002. An Empirical Investigation of Continuous-Time Equity Return Models. Journal of Finance 57: 1239-1284.

Barndorff-Nielsen, O. E., and N. Shephard. 2002. Econometric Analysis of Realized Volatility and Its Use in Estimating Stochastic Volatility Models. Journal of the Royal Statistical Society, Series B 64: 253-280.

Bates, D. S. 2000. Post-'87 Crash Fears in the S\&P 500 Futures Option Market. Journal of Econometrics 94: 181-238.

Bates, D. S. 2006. Maximum Likelihood Estimation of Latent Affine Processes. Review of Financial Studies 19: 909-965.

Bollerslev, T., and H. Zhou. 2006. Volatility Puzzles: A Simple Framework for Gauging Return-Volatility Regressions. Journal of Econometrics 131: 123-150.

Britten-Jones, M., and A. Neuberger. 2000. Option Prices, Implied Price Processes, and Stochastic Volatility. Journal of Finance 55: 839-866.

Broadie, M., M. Chernov, and M. Johannes. 2007. Model Specification and Risk Premia: Evidence from Futures Options. Journal of Finance 62: 1453-1490.

Chernov, M., A. R. Gallant, E. Ghysels, and G. Tauchen. 2003. Alternative Models for Stock Price Dynamics. Journal of Econometrics 116: 225-257.

Chernov, M., and E. Ghysels. 2000. A Study Towards a Unified Approach to the Joint Estimation of Objective and Risk Neutral Measures for the Purposes of Options Valuation. Journal of Financial Economics 56: 407-458.

Christensen, B., and N. Prabhala. 1998. The Relation between Implied and Realized Volatility. Journal of Financial Economics 50: 125-150.

Christoffersen, P., K. Jacobs, and K. Mimouni. 2010. Models for S\&P 500 Dynamics: Evidence from Realized Volatility, Daily Returns, and Option Prices. Review of Financial Studies 23: 2139-2183. 
Corrado, C., and T. Miller. 2005. The Forecast Quality of CBOE Implied Volatility Indexes. Journal of Futures Markets 25: 339-373.

Duffie, D., J. Pan, and K. J. Singleton. 2000. Transform Analysis and Asset Pricing for Affine Jump-Diffusions. Econometrica 68: 1343-1376.

Durbin, J., and S. Koopman. 1997. Monte Carlo Maximum Likelihood Estimation for Non-Gaussian State Space Models. Biometrika 84: 669-684.

Durham, G. B. 2006. Monte Carlo Methods for Estimating, Smoothing, and Filtering One- and Two-Factor Stochastic Volatility Models. Journal of Econometrics 133: 273-305.

Durham, G. B. 2007. SV Mixture Models with Application to S\&P 500 Index Returns. Journal of Financial Economics 85: 822-856.

Durham, G. B., and A. R. Gallant. 2002. Numerical Techniques for Simulated Maximum Likelihood Estimation of Stochastic Differential Equations. Journal of Business and Economic Statistics 20: 297-316.

Elerian, O., S. Chib, and N. Shephard. 2001. Likelihood Inference for Discretely Observed Non-linear Diffusions. Econometrica 69: 959-993.

Eraker, B. 2001. MCMC Analysis of Diffusion Models with Application to Finance. Journal of Business and Economic Statistics 19: 177-191.

Eraker, B. 2004. Do Stock Prices and Volatility Jump? Reconciling Evidence from Spot and Option Prices. Journal of Finance 59: 1367-1403.

Eraker, B., M. Johannes, and N. G. Polson. 2003. The Impact of Jumps in Volatility and Returns. Journal of Finance 58: 1269-1300.

Ferriani, F., and S. Pastorello. 2011. Estimating and Testing Non-Affine Option Pricing Models with a Large Unbalanced Panel of Options. Econometrics Journal 15: 171-203.

Fleming, J., B. Ostdiek, and R. Whaley. 1995. Predicting Stock Market Volatility: A New Measure. Journal of Futures Markets 15: 265-302.

Fouque, J.-P., G. Papanicolaou, and K. Sircar. 2000. Derivatives in Financial Markets with Stochastic Volatility. Cambridge, UK: Cambridge University Press.

Gallant, A. R., and G. E. Tauchen. 1996. Which Moments to Match? Econometric Theory 12: 657-681.

Garcia, R., M.-A. Lewis, S. Pastorello, and E. Renault. 2011. Estimation of Objective and Risk-Neutral Distributions Based on Moments of Integrated Volatility. Journal of Econometrics 160: 22-32.

Ghysels, E., P. Santa-Clara, and R. Valkanov. 2006. Predicting Volatility: Getting the Most Out of Return Data Sampled at Different Frequencies. Journal of Econometrics 131: 59-95.

Jacquier, E., N. G. Polson, and P. E. Rossi. 1994. Bayesian Analysis of Stochastic Volatility Models. Journal of Business and Economic Statistics 12: 371-389.

Jacquier, E., N. G. Polson, and P. E. Rossi. 2004. Bayesian Analysis of Stochastic Volatility Models with Fat-Tails and Correlated Errors. Journal of Econometrics 122: 185-212.

Jiang, G., and Y. Tian. 2005. The Model-Free Implied Volatility and its Information Content. Review of Financial Studies 18: 1305-1342. 
Jones, C. S. 2003. The Dynamics of Stochastic Volatility: Evidence from Underlying and Options Markets. Journal of Econometrics 116: 181-224.

Kim, S., N. Shephard, and S. Chib. 1998. Stochastic Volatility: Likelihood Inference and Comparison with ARCH Models. Review of Economic Studies 65: 361-393.

Kloeden, P., and E. Platen. 1992. Numerical Solution of Stochastic Differential Equations. Berlin: Springer Verlag.

Lewis, A. 2000. Option Valuation under Stochastic Volatility. Newport Beach, CA: Finance Press.

Liesenfeld, R., and J.-F. Richard. 2003. Univariate and Multivariate Stochastic Volatility Models: Estimation and Diagnostics. Journal of Empirical Finance 10: 505-531.

Pan, J. 2002. The Jump-Risk Premia Implicit in Options: Evidence From an Integrated Time-Series Study. Journal of Financial Economics 63: 3-50.

Pastorello, S., V. Patilea, and E. Renault. 2003. Iterative and Recursive Estimation in Structural Nonadaptive Models. Journal of Business and Economic Statistics 21: 449-482.

Pastorello, S., E. Renault, and N. Touzi. 2000. Statistical Inference for RandomVariance Option Pricing. Journal of Business and Economic Statistics 18: 358-367.

Pedersen, A. R. 1995. A New Approach to Maximum Likelihood Estimation for Stochastic Differential Equations Based on Discrete Observations. Scandinavian Journal of Statistics 22: 55-71.

Poteshman, A. M. 1998. “Estimating a General Stochastic Variance Model from Option Prices." Working paper, University of Illinois.

Romano, M., and N. Touzi. 1997. Contingent Claims and Market Completeness in a Stochastic Volatility Model. Mathematical Finance 7: 399-412.

Shephard, N., and M. Pitt. 1997. Likelihood Analysis of Non-Gaussian Measurement Time Series. Biometrika 84: 653-667. 